\title{
A Stochastic Dynamical Model of Arctic Sea Ice $\mathscr{O}$
}

\author{
WoOsOK MOON \\ Department of Applied Mathematics and Theoretical Physics, University of Cambridge, and British \\ Antarctic Survey, Cambridge, United Kingdom \\ J. S. WETTLAUFER \\ Yale University, New Haven, Connecticut, and Mathematical Institute, University of Oxford, Oxford, United Kingdom, \\ and Nordita, Royal Institute of Technology, and Stockholm University, Stockholm, Sweden
}

(Manuscript received 15 March 2016, in final form 16 March 2017)

\begin{abstract}
The noise forcing underlying the variability in the Arctic ice cover has a wide range of principally unknown origins. For this reason, the analytical and numerical solutions of a stochastic Arctic sea ice model are analyzed with both additive and multiplicative noise over a wide range of external heat fluxes $\Delta F_{0}$, corresponding to greenhouse gas forcing. The stochastic variability fundamentally influences the nature of the deterministic steady-state solutions corresponding to perennial and seasonal ice and ice-free states. Thus, the results are particularly relevant for the interpretation of the state of the system as the ice cover thins with $\Delta F_{0}$, allowing a thorough examination of the differing effects of additive versus multiplicative noise. In the perennial ice regime, the principal stochastic moments are calculated and compared to those determined from a stochastic perturbation theory described previously. As $\Delta F_{0}$ increases, the competing contributions to the variability of the destabilizing sea ice-albedo feedback and the stabilizing longwave radiative loss are examined in detail. At the end of summer the variability of the stochastic paths shows a clear maximum, which is due to the combination of the increasing influence of the albedo feedback and an associated "memory effect," in which fluctuations accumulate from early spring to late summer. This is counterbalanced by the stabilization of the ice cover resulting from the longwave loss of energy from the ice surface, which is enhanced during winter, thereby focusing the stochastic paths and decreasing the variability. Finally, common examples in stochastic dynamics with multiplicative noise are discussed wherein the choice of the stochastic calculus (Itô or Stratonovich) is not necessarily determinable a priori from observations alone, which is why both calculi are treated on equal footing herein.
\end{abstract}

\section{Introduction}

The advantages of simple deterministic theories of climate, such as clear assessment of stability and feedbacks, were evidently first recognized in the context of energy flux balance models independently by Budyko (1969) and Sellers (1969). Such approaches reveal key issues, such as the role of albedo feedback in planetary climate, the potential coexistence of multiple climate states under ostensibly the same forcing conditions, and the nature of the transition of mean states between them. Important early extensions of the original models

Supplemental information related to this paper is available at the Journals Online website: http://dx.doi.org/10.1175/ JCLI-D-16-0223.s1.

Corresponding author: Woosok Moon,wooon@bas.ac.uk including a form of meridional heat transfer are still analytically solvable and can be used to assess the stability of high-latitude ice caps under varying climatic conditions (e.g., Held and Suarez 1974; North 1975; North and Cahalan 1981). The inclusion of additional physics, such as diffusive-type transport, can decrease the sensitivity of solutions relative to the simplest models (e.g., Lindzen and Farrell 1977) or bring out more stable solutions (e.g., Rose and Marshall 2009) while sacrificing the ability to find analytical solutions. Indeed, Lindzen and Farrell (1977) point out that there is no a priori compelling reason to assume that simple models with transport are superior than the BudykoSellers type of model. In addition, solely deterministic models cannot capture the role of variability.

In contrast, fully coupled climate models attempt to deterministically treat all of the processes in the climate system and to thereby capture the spatiotemporal 
structure of the atmosphere-land-ocean system. Nonetheless, the inevitable complexity accompanying such treatments often precludes a clear identification of cause and effect in the absence of independent (e.g., observational) information. However, this may be due to a confluence of real feedbacks and highly parameterized processes conspiring to obfuscate a variety of key interactions. Moreover, in the Arctic projections vary widely among the IPCC models regarding the degree of ice loss through 2100 (see, e.g., Fig. 3 of Eisenman et al. 2011).

Stochastic climate models reside in a conceptual region between these two approaches having been introduced to develop a statistical understanding of the climate system or its subsystems (e.g., Hasselmann 1976; North and Cahalan 1981; Benzi et al. 1981; Nicolis and Nicolis 1981; Saltzman 2002; Dijkstra 2013). In general the stochastic approach provides an important niche between solely deterministic low-order models, which were not designed to treat high-frequency variability, and complex fully coupled climate models. In the spirit of the Langevin theory of Brownian motion, stochastic models typically consist of an underlying deterministic model augmented by stochastic forcing. The deterministic dynamics tends to embody the core physics of the system of note, and the stochastic forcing captures the short-time-scale processes that modify the deterministic dynamics. Solutions of stochastic models provide the statistics underlying the variability that characterizes the interplay between the slow and fast dynamics. This interplay introduces a complexity that can yield dynamics that are qualitatively different from simple deterministic models alone, while providing a richness that is seen in climate models, and yet still within a framework amenable to analysis.

The evolution of the air-sea-ice system has long been recognized as being a stochastic system (e.g., Lemke 1986, and references therein), and here we focus on a stochastic energy balance model of Arctic sea ice. During the satellite era, in which we have high-fidelity measurements of the extent of the ice cover, there have been significant decreases in volume and extent (see, e.g., Kwok and Untersteiner 2011; Meier et al. 2014, and references therein). While both the observational record (Agarwal et al. 2012) and climate model simulations (Eisenman et al. 2011) exhibit substantial variability on multiple time scales, it is clear that the mean minimum ice extent is decaying, which has stimulated the question of whether and when a seasonal ice state-no ice in the summer-may appear. Importantly, satellite data reveal that the nature of the noise itself is multifractal (Agarwal et al. 2012), and thus given the prominence of variability in the observational record, a central question concerns how noise will impact the potential transitions in the state of the ice cover. Because the observations show the complexity of the noise structure, and there is no a priori evidence for a "correct" theoretical treatment (e.g., additive vs multiplicative) (Moon and Wettlaufer 2014), stochastic models must explore the influences of different but rigorous treatments.

The response of the seasonal cycle of Arctic sea ice thickness to climate was first reproduced quantitatively in the thermodynamic model of Maykut and Untersteiner (1971). The essence of this work has been captured more recently in several simpler models developed in the spirit of Budyko (1969) and Sellers (1969) to assess the question of the transitions between perennial and seasonal ice and ice-free states (Thorndike 1992; Eisenman and Wettlaufer 2009). These approaches reproduce the observed season cycle of ice thickness, and we use that of Eisenman and Wettlaufer (2009) as the deterministic backbone of our stochastic model for the following reasons. First, we have assessed in detail the stability of the deterministic steady states of this model and found the two key competing factors that dominate the response time scales (Moon and Wettlaufer 2011). In particular, the response time scales are governed by the destabilizing ice-albedo feedback and the stabilizing longwave radiative energy loss, which reflects the well-known fact that thin ice grows more rapidly than thick ice (see, e.g., Fig. 2 of Moon and Wettlaufer 2011). Second, we have developed a perturbative framework of determining analytic solutions of the stochastic model that capture the key statistical moments of perennial ice states (Moon and Wettlaufer 2013). Third, the approach reveals a "memory effect" whereby the intrinsic nonlinearity, asymmetry, and stability characteristics of the interaction between the deterministic backbone and the noise provide an interpretive framework of cause and effect, along with their response time scales. Finally, numerical solutions to this model provide unique visualization of stochastic paths and probability density functions (PDFs) under the influence of increased greenhouse gas forcing $\Delta F_{0}$. This extends our analysis beyond the range available to our perturbative framework to allow examination of the dynamics of seasonally varying states.

Because we can physically rationalize using both additive and multiplicative noise forcing on the same deterministic backbone, we present both here, although we note this makes for a rather weighty presentation. In particular, as discussed in detail in section $2 \mathrm{c}$ below, in the case of multiplicative noise we give the both stochastic calculi-Itô and Stratonovich-equal weighting and thereby compare simulations using both. As we increase $\Delta F_{0}$ the stochastic stability of the system is examined in light of the expectations from the deterministic dynamics - transitions in the ice state are "blurred" by the variability in the stochastic paths. The structure of the paper is as follows. In the next section we 
describe the stochastic model and the numerical scheme. We analyze the steady state stochastic solutions (viz., stochastic paths, PDFs, and statistical moments) in section 3. The overall dynamics is put in the framework of an "ice potential," which is a seasonally evolving potential encoding the competition between stabilizing and destabilizing effects and how these change with $\Delta F_{0}{ }^{1}{ }^{1}$ In this sense it is heuristically like an Ornstein-Uhlenbeck process, in a time-dependent potential, although we note that the deterministic backbone is nonlinear and nonautonomous. We summarize and discuss the findings in section 5 .

\section{Stochastic sea ice model and numerical methods}

\section{a. Stochastic Arctic sea ice model}

The stochastic Arctic sea ice model that forms the basis of our simulations has been described previously [Eqs. (2) or (66) of Moon and Wettlaufer 2013], but to ensure that this paper is self-contained we summarize it here. The system is governed by a dimensionless Langevin equation written as

$$
d E=a(E, t) d t+b(E, t) \circ d W,
$$

where the first term on the right-hand side represents the deterministic backbone of the stochastic model, which is equivalent to that of Eisenman and Wettlaufer (2009), and the second term treats the stochastic forcing where $d W$ represents a Wiener process, with $\circ$ denoting the Stratonovich interpretation of the noise as opposed to the Itô interpretation, discussed below in section $2 \mathrm{c}$.

The energy $E$ is defined as the amount of latent heat stored in a layer of ice of thickness $h$ or in the ocean mixed layer if the ice vanishes. The convention used is that ice is present (absent) when $E$ is negative (positive). The deterministic energy balance term $a(E, t)$ is

$$
\begin{aligned}
a(E, t) \equiv & {[1-\alpha(E)] F_{S}(t)-F_{0}(t)-F_{T}(t) T(t, E) } \\
& +\Delta F_{0}+F_{B}+\nu \mathcal{R}(-E),
\end{aligned}
$$

where

$$
\alpha(E)=\frac{\alpha_{\mathrm{ml}}+\alpha_{i}}{2}+\frac{\alpha_{\mathrm{ml}}-\alpha_{i}}{2} \tanh \left(\frac{E_{0}}{L_{i} h_{\alpha}} E\right) \text { and }
$$

Here, $\alpha(E)$ is the surface albedo and $T(t, E)$ the surface temperature. The fraction $1-\alpha(E)$ of the incident shortwave radiation $F_{S}(t)$ absorbed at the surface is modeled with an albedo function based on the BeerLambert law of exponential attenuation of radiative intensity with depth using a characteristic ice thickness $h_{\alpha}=0.5 \mathrm{~m}$ for extinction. It captures the transition from perennial sea ice albedo $\left(\alpha_{i}=0.68\right)$ to ocean albedo $\left(\alpha_{\mathrm{ml}}=0.2\right)$ and in this manner models the ice-albedo feedback-it is clearly operative when the ice thickness approaches $h_{\alpha}$. The core deterministic term $\alpha(E, t)$ describes the energy flux balance at the atmosphere-ice (ocean) interface where we calculate the surface temperature $T(t, E)$. Quantitatively, this balance is dominated by incoming shortwave radiation, outgoing

\footnotetext{
${ }^{1}$ The core dynamics are studied with the dimensionless version of the model, but throughout this paper when we refer to values of $\Delta F_{0}$ they are understood to carry units of $\mathrm{W} \mathrm{m}^{-2}$.
}

longwave radiation, and the conductive heat flux through sea ice. During winter, the principal stabilizing mechanism is associated with how longwave radiative loss drives ice growth-thin ice grows more rapidly than thick ice (Stefan 1889). During summer, the principal destabilizing mechanism is the ice-albedo feedback, which becomes more prevalent when ice thins and approaches $h_{\alpha}$. The observed average annual export of about $10 \%$ (Kwok et al. 2004) acts as a constant sink of energy, here represented by $\nu \mathcal{R}(-E)$, where $\nu=0.1$. The ramp function is $\mathcal{R}(x \geq 0)=x$ and $\mathcal{R}(x<0)=0$, which captures the transition between freezing and melting states and the fact that sea ice export occurs only when sea ice is present.

A more detailed description of the derivation of $\alpha(E, t)$, including the incorporation of the various surface fluxes, the meridional heat flux due to large-scale atmospheric motions and the radiative transfer model is described in Eisenman and Wettlaufer (2009). The stability of the deterministic model and the core competition between the destabilizing ice-albedo feedback and the stabilizing 
longwave loss at the ice surface is detailed in Moon and Wettlaufer (2011), which forms an important foundation for our interpretation of the stochastic dynamics.

Recently, Wagner and Eisenman (2015) suggested that the inclusion of a latitudinal variation in a deterministic single column model can substantially change the structure of the bifurcation diagram, thereby indicating that such complexities demarcate a model's ability to treat realistic behavior. However, it is a basic result in the theory of dynamical systems (Tredicce et al. 2004) that, even in the simplest of models, when one constructs a bifurcation diagram with a slowly time-varying control parameter rather than a constant value, substantially different results are obtained. Hence, both complexity and the basic mathematical treatment are important.

\section{$b$. The role of the sea ice-albedo feedback}

The most important process controlling the statistics of the stochastic solutions is the ice-albedo feedback. The solution behavior is influenced by the asymmetric (signed) response of the ice to a perturbation associated with the dependence of albedo upon thickness as depicted in Fig. 1. The magnitude of the feedback depends upon the sensitivity of the albedo to a perturbation, which begins to become effective when $h \approx h_{\alpha}$. The asymmetry is demonstrated for two ice states, $\mathrm{A}$ and $\mathrm{B}$. State A describes ice during summer when $\Delta F_{0} \approx 19.0$. Here, a positive (negative) perturbation will be more (less) effective in changing the ice energy $E$ because of the sharp decrease (small change) of the albedo. State $\mathrm{B}$ describes ice during summer when $\Delta F_{0} \approx 20.0$ and the sea ice is very thin. In contrast to state A, a negative (positive) perturbation will be more (less) effective in changing the ice energy $E$ because of the sharp increase (small change) of the albedo. Hence, very small changes in $\Delta F_{0}$ near this transition can generate highly variable stochastic paths. This must be understood as a stochastic effect rather than a trend associated with increasing $\Delta F_{0}$; the key point is that the variability increases with greenhouse gas forcing. This process is particularly important for understanding the solution statistics near the transition from perennial to seasonally varying ice states.

\section{c. Numerical method}

There are a wide variety of numerical methods used to solve stochastic ordinary differential equations (e.g., Kloeden and Platen 1992). Most such methods rely upon a Taylor expansion, within either the Itô or Stratonovich calculus framework. The order of numerical methods is determined by the convergence of either 1) the path of the solution itself (strong convergence) or 2) the statistical moments (weak convergence). The inclusion of one higher-order term in the Taylor expansion

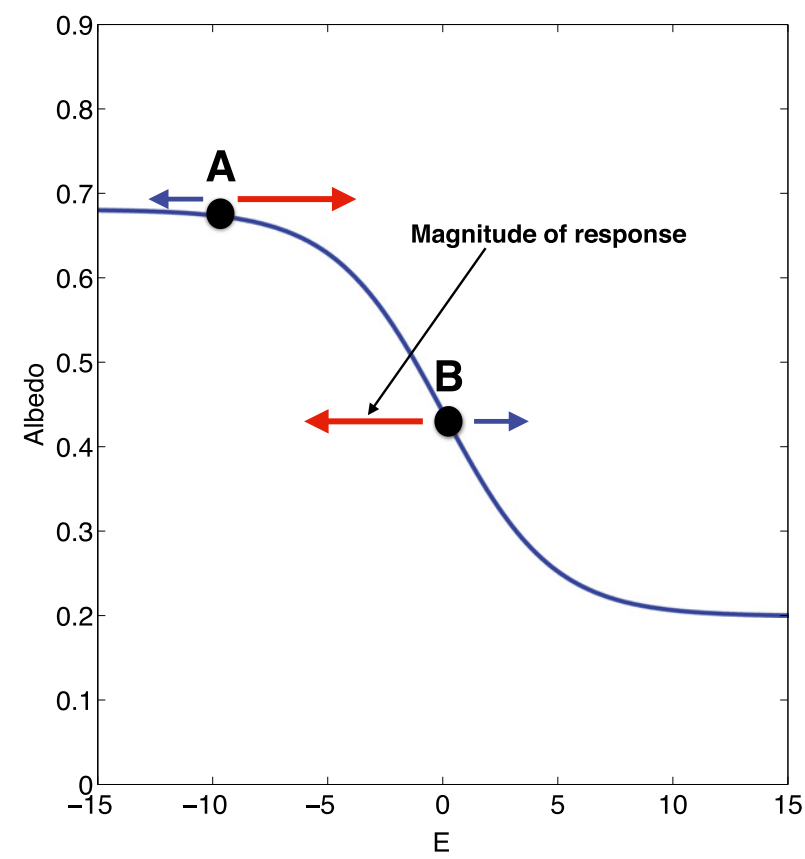

FIG. 1. Schematic diagram showing the relationship between magnitude of the response of sea ice energy (thickness) and the albedo. States A and B represent two examples describing the asymmetric response of sea ice to a given (signed) perturbation. State A describes ice during summer when $\Delta F_{0} \approx 19.0$ and the icealbedo feedback starts to operate. A positive perturbation will be more effective in changing the ice energy $E$ as a result of the sharp decrease of the albedo. State $\mathrm{B}$ is relevant when $\Delta F_{0} \approx 20.0$ and the sea ice is very thin during summer. Conversely to state $\mathrm{A}$, a negative perturbation will be more effective in changing the ice energy $E$ as a result of the sharp increase of the albedo.

increases the numerical order by 0.5 (1.0) in the sense of the strong convergence (weak convergence). For many cases, order 1.0 (2.0) methods in strong (weak) convergence are sufficient. Here, we use a weak order 2.0 approach based upon the Runge-Kutta method of Tocino and Vigo-Aguiar (2002). The discrete form of the Eq. (1) is written as follows:

$$
\begin{aligned}
E_{n+1}= & E_{n}+\frac{1}{2}\left(k_{0}+k_{1}\right) \Delta+\frac{1}{4}\left(2 s_{0}+s_{1}+s_{2}\right) \Delta W_{n} \\
& +\frac{1}{4}\left(s_{2}-s_{1}\right)\left[\sqrt{\Delta}-\frac{\left(\Delta W_{n}\right)^{2}}{\sqrt{\Delta}}\right],
\end{aligned}
$$

where

$$
\begin{aligned}
& k_{0}=\bar{a}\left(E_{n}, t_{n}\right), \\
& s_{0}=b\left(E_{n}, t_{n}\right), \\
& k_{1}=\bar{a}\left(E_{n}+k_{0} \Delta+\Delta W_{n} s_{0}, t_{n}+\Delta\right), \\
& s_{1}=b\left(E_{n}+k_{0} \Delta+\sqrt{\Delta} s_{0}, t_{n}+\Delta\right), \quad \text { and } \\
& s_{2}=b\left(E_{n}+k_{0} \Delta-\sqrt{\Delta} s_{0}, t_{n}+\Delta\right) .
\end{aligned}
$$


If the stochastic model is interpreted within the framework of Itô calculus then $\bar{a}(E, t) \equiv a(E, t)$, whereas in the framework of Stratonovich calculus $\bar{a}(E, t) \equiv$ $a(E, t)+1 / 2 b(E, t) \partial_{E} b(E, t)$. This transformation between the two forms of stochastic calculus was introduced by Wong and Zakai (1965), and there are more pedagogical discussions of the mathematical background and geophysical applications found in Doering (2016) and Dijkstra (2013). The time step is $\Delta$ and $\Delta W_{n}$ is a Gaussian variable whose mean and standard deviation are 0 and $\sqrt{\Delta}$, respectively. Using this method and converting to dimensional time the system reaches a steady state in 20 years. To generate ensemble statistics, we repeat the simulation using different values of $\Delta W_{n}$ and different values of $\Delta F_{0}$. The baseline numerical analysis uses $10^{6}$ ensemble simulations with a $10^{-6}$ yr time step and a noise intensity of 0.05 .

\section{ADDITIVE AND MULTIPLICATIVE NOISE STRUCTURE}

Clearly, the simplest form of additive noise transforms the function $b(E, t)$ on the right-hand side of Eq. (1) into a (typically small) constant $b$. This is generally referred to as constant additive noise. However, since we are dealing with a deterministic dynamics that is time periodic, there are a range of possible additive noise scenarios that can be treated. We describe our approach presently.

We introduce multiplicative noise through variability in sea ice export, which we can ascribe to the observation that the geostrophic wind field that drives ice motion can be treated as a Gaussian random field (Thorndike 1982; Agarwal and Wettlaufer 2017). To include the effect of fluctuations upon the sea ice export, we introduce a random variable as $\nu=\nu_{0}+\sigma \xi(t)$, where the constant value $\nu_{0}=0.1$ becomes that from the deterministic dynamics and $\xi(t)$ is related to the Weiner process as $\xi(t)=d W / d t$. Hence, $b(E, t)$ of Eq. (1) becomes $\sigma \mathcal{R}(-E)$ and we can rewrite the stochastic model as

$$
d E=a(E, t) d t+\sigma \mathcal{R}(-E) \circ d W,
$$

where the noise amplitude $\sigma$ is small relative to unity (for our numerical studies it is set to 0.05$)$, and the deterministic term $a(E, t)$ is as in Eq. (2) but with $\nu \rightarrow \nu_{0}=0.1$.

It is important to note that even in well-studied nonlinear systems, the mathematical and physical interpretation of multiplicative noise depends upon the choice of stochastic calculus, and there are subtle issues arising even in the simplest form of additive noise. A core difference between the calculi resides in the freedom to choose the value of the integrand in a subinterval of the Riemann sum. For example, Itô calculus is often preferred because it preserves the Martingale property, wherein the expectation value of any time-dependent quantity depends solely upon the present value. Although this approach has many practical numerical advantages, the usual rules of calculus are not obeyed, whereas this is not the case with Stratonovich calculus. In this setting, the major difference between Stratonovich and Ito calculus is the shift of the mean value due to the accumulation of noise forcing. Here, we will consider both perspectives numerically through simultaneous treatment of the statistical moments and the stochastic paths.

Wong and Zakai (1965) argued that there is no realworld system in which perfect white noise exists. Thus, Brownian motion $x(t)$ approximates a description $x_{n}$ $(t)$ that is continuous with at least a piecewise continuous derivative. By showing that $x_{n}(t) \rightarrow x(t)$ as $n \rightarrow \infty$ they recovered Stratonovich calculus. Accordingly, the choice of stochastic calculus resides in the characteristics of the noise and continuity arguments (Moon and Wettlaufer 2014). On one hand, in statistical physics white noise is typically defined through a $\delta$-autocorrelation, and it is also suggested that this definition is equivalent to Stratonovich calculus (Risken 1984). Thus, the use of white noise to approximate high-frequency processes in systems observed over much longer time scales is often argued to be within the purview of Stratonovich calculus. On the other hand, in finance and biology, most of the highfrequency processes are assumed to be discrete, and hence the above arguments may not be applied. Thus, Itô calculus is assumed to be appropriate (Turelli 1977; Shreve et al. 2004), thereby maintaining the Martingale property. In terms of overall separation of time scales, there is no conceptual distinction between the statistics of water molecules colliding pollen grains and the trading equities (or the like). Hence, the question remains if, how, and when it is appropriate to use continuity considerations as a core criterion to choose either of the calculi being discussed here.

We believe the choice of which stochastic calculus should be used for a particular set of physical processes is more complicated than the above. For example, in building a mathematical model it is common to ignore the influence of high-frequency processes on the deterministic dynamics, although we know there are situations when this is a poor assumption, such as in the presence of inertial (Kupferman et al. 2004) or feedback (Pesce et al. 2013) effects. Indeed, when Kupferman et al. (2004) studied systems with multiplicative colored noise and inertia they found that if the correlation time of the noise is faster (slower) than the relaxation time, 
this leads to the Itô (Stratonovich) calculus form of the limiting stochastic differential equation. Similarly, Itô calculus is invoked to interpret experiments wherein the time delay of the feedback is much larger than the noise correlation time (Pesce et al. 2013). Hence, there is an experimental demonstration that the choice of the stochastic calculus is not necessarily a priori determinable from observations alone. Indeed, even taking the white noise limit of a colored noise process, which leads to Itô calculus, this is a deliberate choice, which is often made for numerical reasons-principally the appeal of the aesthetics of the standard forward Euler scheme (Doering 2016).

For these reasons, and those found in a more detailed discussion (Moon and Wettlaufer 2014), we take an agnostic approach and treat the two calculi with equal weighting. Although the noise structure of Arctic sea ice can be quantified from observations (Agarwal et al. 2012), the choice of stochastic calculus cannot be deduced from them. Hence, we believe that comparing the stochastic solutions from the two calculi will be beneficial to those trying to implement stochastic models in a variety of contexts.

For the most general multiplicative noise case $\sigma \mathcal{R}[-E(t)] \xi(t)$, with $|\sigma| \ll 1$, we have a theoretical framework with which to compare our numerical results (Moon and Wettlaufer 2013). Because the theoretical framework is perturbative, several more cases are then naturally structured for comparison. We define seasonally varying additive noise (SVA) when the noise magnitude is $\sigma \mathcal{R}\left[-E_{S}(t)\right]$, where $E_{S}(t)$ is the deterministic steady-state solution and hence the noise is additive but time varying with the seasonal cycle. The constant additive noise (CA) case is a natural limit of the seasonally varying additive noise case and has noise amplitude $\sigma \overline{\mathcal{R}\left[-E_{S}(t)\right]}$, where the overbar is the seasonal time average of $E_{S}$ $(t)$. The difference between these two cases reveals the impact of seasonally varying noise magnitude.

It is prudent to deal with all of these cases because the first-order perturbative solution is equivalent to that with seasonally varying noise, and the effect of multiplicative noise upon the steady-state stochastic solutions does not appear until the second order. Therefore, we will compare the full model described by Eq. (11) with the seasonally varying noise case to reveal the bare effect of multiplicative noise.

Four similar but systematically different cases will be analyzed and compared. We first compare the CA and the SVA cases and then the two different stochastic calculi, Itô (IM) and Stratonovich (SM), where the M denotes multiplicative. This allows us to compare and contrast the role of different classes of noise forcing.

\section{Results: Additive noise cases}

In this section we describe a large suite of simulations of this model using the numerical method explained in the previous section. We obtain many stochastic realizations and generate ensemble statistics. As noted above, the amplitude of the constant additive noise forcing is fixed at 0.05 for all simulations. One advantage of the numerical simulations over our solely analytical method is the ability to observe the evolution of a specific stochastic realization and to directly construct a PDF for a given type of noise forcing. Moreover, we can explore the stochastic solutions over a range of $\Delta F_{0}$ in which our stochastic perturbation method cannot be applied, although we will still compare the numerical solutions to the analytic solutions over their range of validity (Moon and Wettlaufer 2013). We thereby extend our understanding and analysis to the seasonally varying states, where stochastic effects are particularly important.

Depending on the geometric structure of the deterministic backbone of the model in the vicinity of the steady-state solution, and the nature of the noise forcing, the stochastic solution will exhibit dispersion relative to the deterministic solution, giving rise to asymmetry in stochastic realizations.

\section{a. Perennial ice states}

Deterministic perennial ice states exist under greenhouse gas forcing $\Delta F_{0}$ up to approximately 20 when a continuous transition to a seasonally varying state occurs, whereas beyond approximately 23 the seasonal ice vanishes in a saddle-node bifurcation to a perennial ice-free state (see Fig. 3 of Eisenman and Wettlaufer 2009). Thus, as a first example, in Fig. 2 we show stochastic realizations as $\Delta F_{0}$ grows from 10 to 18 . It is noticeable that even with the same noise forcing, the spread of the stochastic realizations increases with $\Delta F_{0}$, with some realizations exhibiting seasonal ice states under forcing in which the deterministic state has perennial ice.

As described previously (Moon and Wettlaufer 2013), the stochastic model can be represented in an approximate form near the deterministic solution $E_{S}$ $\left(t, \Delta F_{0}\right)$ as follows. If we let $E(t)=E_{S}(t)+\eta$, where $\eta$ is the departure from the deterministic solutions and is written as

$$
\frac{d \eta}{d t}=c(t) \eta+d(t) \eta^{2}+\sigma \xi
$$

where $c(t) \equiv \partial a(E, t) /\left.\partial E\right|_{E=E_{S}}$ and $d(t) \equiv 1 / 2 \partial^{2} a(E, t) /$ $\left.\partial E^{2}\right|_{E=E_{S}}$, and $a(E, t)$ is that from Eq. (1). Here, we 


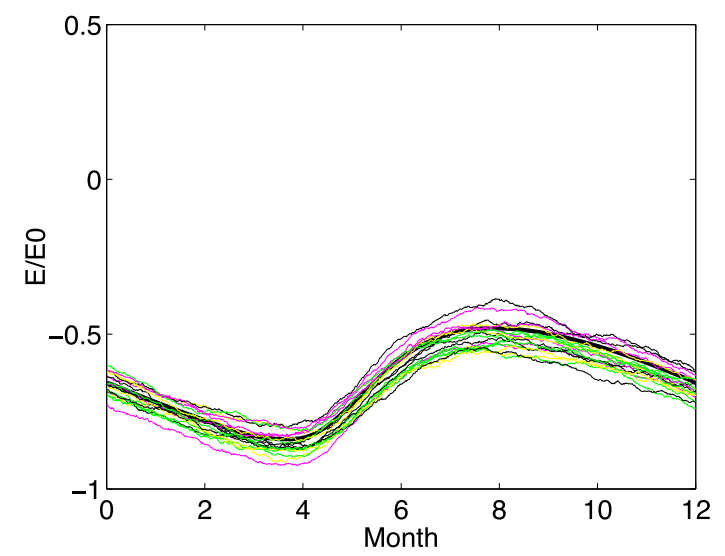

(a)

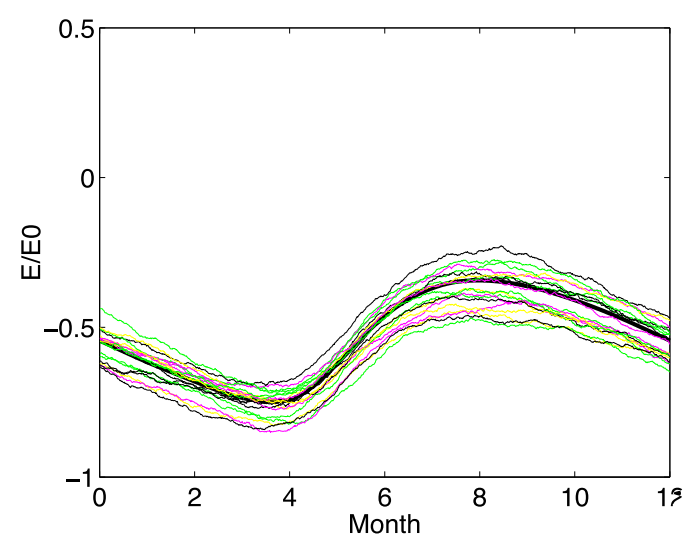

(b)

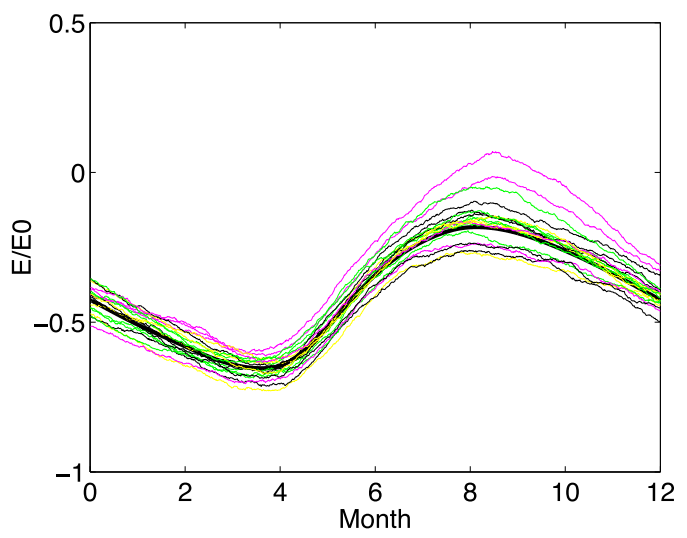

(c)

FIG. 2. Several realizations of the seasonal cycle of the stochastic solutions with three different values of $\Delta F_{0}$ : (a) 10.0, (b) 14.0, and (c) 18.0. The thick black lines represent deterministic stable seasonal cycles of sea ice thickness. The other lines show different realizations of the stochastic solutions.

introduce the ice potential $V(\eta, t) \equiv-(1 / 2) c(t) \eta^{2}-$ $(1 / 3) d(t) \eta^{3}$ to rewrite Eq. (12) as

$$
\frac{d \eta}{d t}=-\frac{\partial}{\partial \eta} V(\eta, t)+\sigma \xi
$$

The interpretation of both the analytic and numerical solutions is facilitated by examining the structure of the potential $V(\eta, t)$, which reflects the geometry of the deterministic solutions. $^{2}$

In an autonomous dynamical system, only a single potential controls the influence of a given perturbation. Here, we have a periodic nonlinear nonautonomous dynamical system, which is much more complicated because the potential evolves continuously. The

\footnotetext{
${ }^{2}$ Note that because $\eta$ is an energy variable we discuss $V(\eta, t)$ and $V(E, t)$ interchangeably.
}

instantaneous stability of the system is reflected in the shape of $V$ (concave or convex). However, as shown in Fig. 3, the potentials are not symmetric about the deterministic solutions. The response of the system to a perturbation is dependent on its sign and is proportional to the slope of $V$. This is understood as being due to the nonlinearity in $a(E, t)$, which is reflected in $d(t)$. The essence of the nonlinearity is that at a given time the response time scale is dependent upon the state of the system-the sea ice thickness.

The potentials during the cold periods shown (February and November) are concave (Fig. 3). As $\Delta F_{0}$ increases the concave minima deepen (cf., e.g., $\Delta F_{0}=10.0$ and 14.0). Physically, this reflects the long-understood phenomenon that thinner ice grows faster than thicker ice (Stefan 1889). Heat conduction is proportional to $\Delta T / h$, where $\Delta T$ is the temperature difference between the top and the bottom of sea ice of thickness $h$. Because the growth rate of the ice depends on how efficiently the 


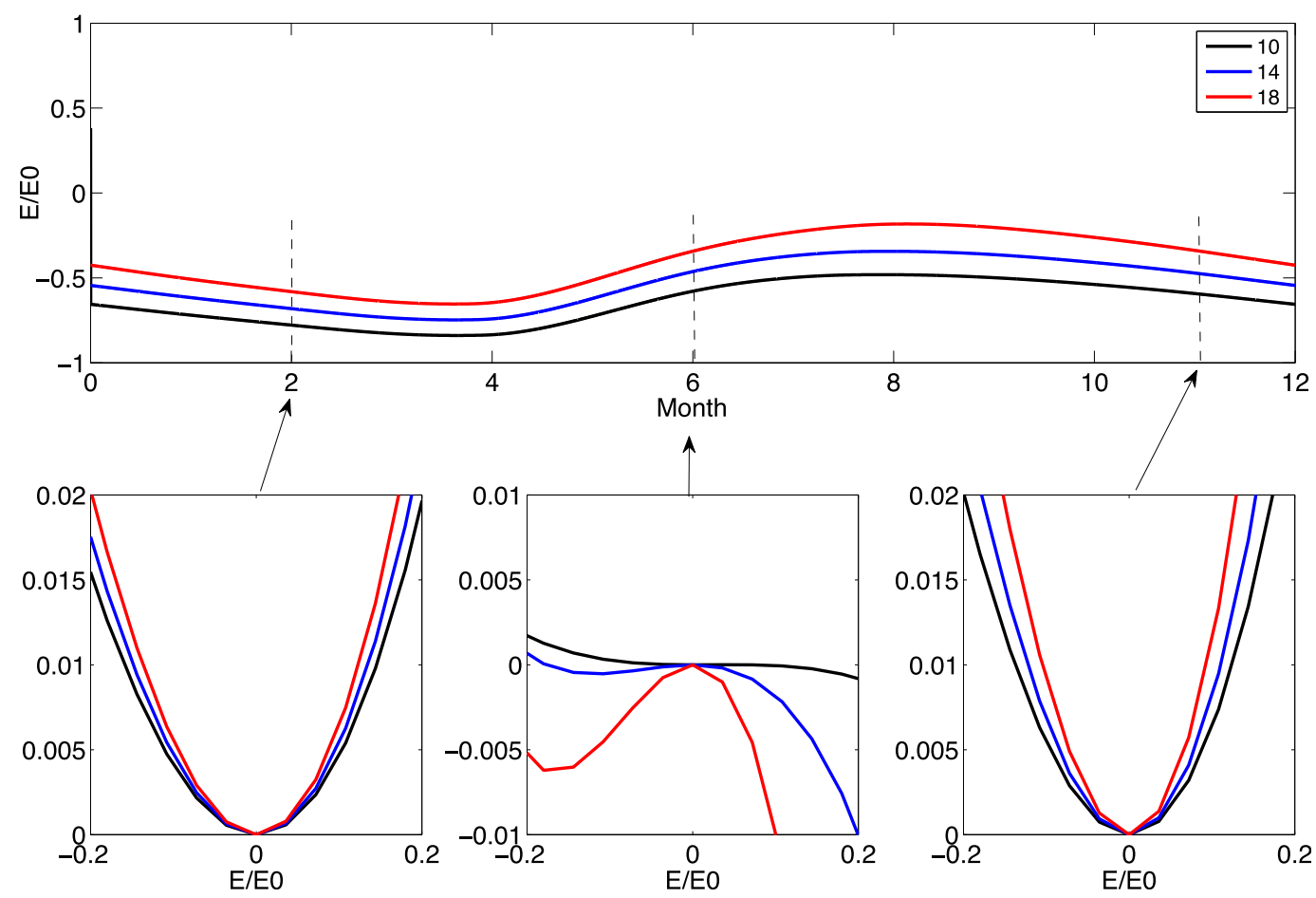

FIG. 3. (top) The stable periodic steady-state solutions at three different values of $\Delta F_{0}: 10.0$ (black), 14.0 (blue), and 18.0 (red). (bottom) The potential $V(E, t)$ for the same values of $\Delta F_{0}$ in (left) February, (center) June, and (right) November. The sign of $E / E_{0}$ is the same as the sign of $\eta$.

latent heat and the oceanic heat flux can be conducted through it, for the same surface heat balance thin ice grows faster than thick ice. In contrast, near zero the potentials during the summer are convex, and the asymmetry about the origin becomes larger as $\Delta F_{0}$ increases. For $\Delta F_{0}=10.0$, the potentials in June or July are almost flat near the origin. However, when $\Delta F_{0}=$ 18.0, the potentials at the same time are convex and asymmetric with the magnitude of the slope being larger for $\eta>0\left(E / E_{0}>0\right)$. The origin of this behavior is that the ice-albedo feedback is more sensitive as the ice thins and the magnitude of the energy $|E|$ decreases.

The potentials shown in Fig. 3 demonstrate the overall seasonal variation. The potentials for November and February reflect the longwave stabilization during winter, suppressing the effects of perturbations, and those for June show that the effect of the icealbedo feedback is to amplify the magnitude of a perturbation. These two main processes combine with the effects of stochastic forcing determine the steady-state stochastic solutions of the model. We stress that, although we show several examples of $V(\eta, t)$ in Fig. 3, the potential changes continuously in time thereby impacting the stochastic paths.

As we have described previously (Moon and Wettlaufer 2013), the steady-state stochastic solutions are determined by the cumulative influence of the potentials in the time domain, which is scaled by the response time of the deterministic solutions. This rectification was referred to as the memory effect. The stochastic paths change continuously as the potential $V(\eta, t)$ changes, exhibiting a clear seasonality of trajectories. At the end of the winter (summer), the stochastic paths are more concentrated (widely distributed) about $E / E_{0}=0$, reflecting the deterministic physics of longwave radiative stabilization and the destabilizing ice-albedo feedback. There is little difference between $\Delta F_{0}=10.0$ and 14.0, but as $\Delta F_{0}$ increases to 18.0 , the stochastic paths are more widely distributed, as can be seen in the supplementary material. In particular, the variability of the paths at the end of summer exhibit a clear maximum (Fig. 4), which is due to the combination of the increasing importance of the ice-albedo feedback and the associated memory effect accumulating a signal from early spring to late summer.

The seasonality of the PDFs can be understood in terms of the memory effect. For example, the PDFs in March have a sharp peak near the deterministic steadystate solution $\left(E / E_{0}=0\right)$. This is explained by the concave shape of the potentials from September to February, which ensures that perturbations converge to $E / E_{0}=0$. Conversely, the destabilizing effect of the ice-albedo 


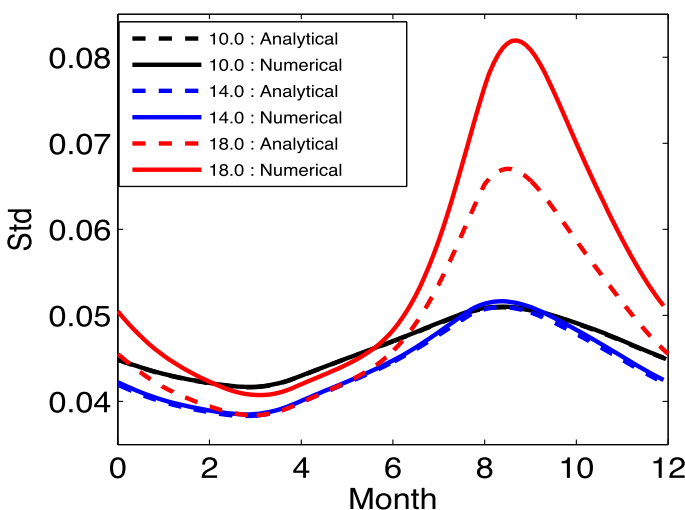

(a)

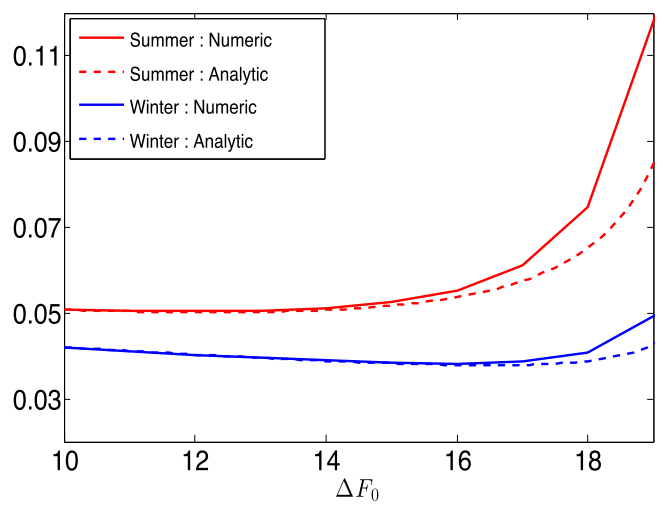

(b)

FIG. 4. Comparison between analytical (dashed lines) and numerical (solid lines) solutions for (a) the standard deviation of the seasonal cycle for three different values of $\Delta F_{0}$ shown in the legend and (b) as a function of $\Delta F_{0}$.

feedback is cumulative, beginning in April or May and reaching a maximum by the end of summer. At any instant the stochastic solutions embody the delayed effect of these competing destabilizing and stabilizing processes. For example, while the ice-albedo feedback begins in April or May and is active all summer, the PDFs are not significantly positively skewed until the end of summer. This reflects the memory effect.

\section{VALIDITY OF ANALYTICAL SOLUTIONS}

Previously we calculated perturbatively the first three moments using (in part) the noise amplitude $\sigma$ as a small parameter, finding the standard deviation at $O(\sigma)$ and the mean and skewness at $O\left(\sigma^{2}\right)$ (Moon and Wettlaufer 2013). In Fig. 4, we compare the analytic solutions with the numerical solutions for three different values of $\Delta F_{0}$. The match between two solutions is excellent for lower values of $\Delta F_{0}$, but, as expected, the deviation grows with $\Delta F_{0}$. The deviation between the theory and the numerical solutions that starts to appear as $\Delta F_{0}$ increases is due to the fact that, at a first order, the perturbative solutions fail to include the asymmetric effects associated with the ice-albedo feedback as seen in the structure of the seasonally varying potentials.

The essence of the perturbative theory is that to first approximation the PDF is Gaussian with a mean equal to that of the deterministic steady-state solutions and the standard deviation changes periodically depending on the time-dependent state of stability as reflected in the ice potential. The deviation of the stochastic means from the deterministic solutions and the skewness appear at the second order. The basic behavior of the solutions at each order is determined by the interplay between the stability of the ice, the nonlinearly induced asymmetry in the response, and the intensity of the noise forcing. In particular, the analytic solutions nicely describe the memory effect in the form of a delayed integral, which is used to interpret the seasonality of the stochastic solutions. The memory effect combines the cumulative influence of the interaction between the statistical fluctuations over the seasonal cycle and the stabilizing and destabilizing processes embodied in the deterministic ice potential, which is also reflected in the Floquet exponents of the deterministic solutions.

The increasingly non-Gaussian behavior as $\Delta F_{0}$ increases demonstrates the limitations of the analytic method. The reason for this deviation is clear; the method is based upon small-amplitude noise forcing and thus implicitly assumes that the behavior of stochastic paths is mainly controlled by the stability and the asymmetry embodied in the deterministic solutions. Such behavior depends principally upon the characteristics inherited from the deterministic solutions rather than the stochastic paths. However, when $\Delta F_{0}$ is large, thin ice is particularly sensitive to the ice-albedo feedback. Therefore, the stochastic paths are not only affected by the stability and the asymmetry of the deterministic dynamics but they are also highly dependent upon the noise-induced variability. For example, positive stochastic forcing during summer is magnified because of the ice-albedo feedback and then significantly damped during winter by the intensification of the longwave stabilization. This leads to a larger response of the statistical moments relative to the analytic solutions.

Moving out of the range of validity of the analytical framework, in the next section we will study the regime of $\Delta F_{0}$ where we have stable seasonally varying states. However, we can still rely on the theory to interpret solutions within the context of the behavior of the local ice potentials.

\section{b. Seasonal ice states}

According to the deterministic theory, the transition from a perennial ice state to a seasonally varying state (with an ice-free summer) is continuous and reversible 


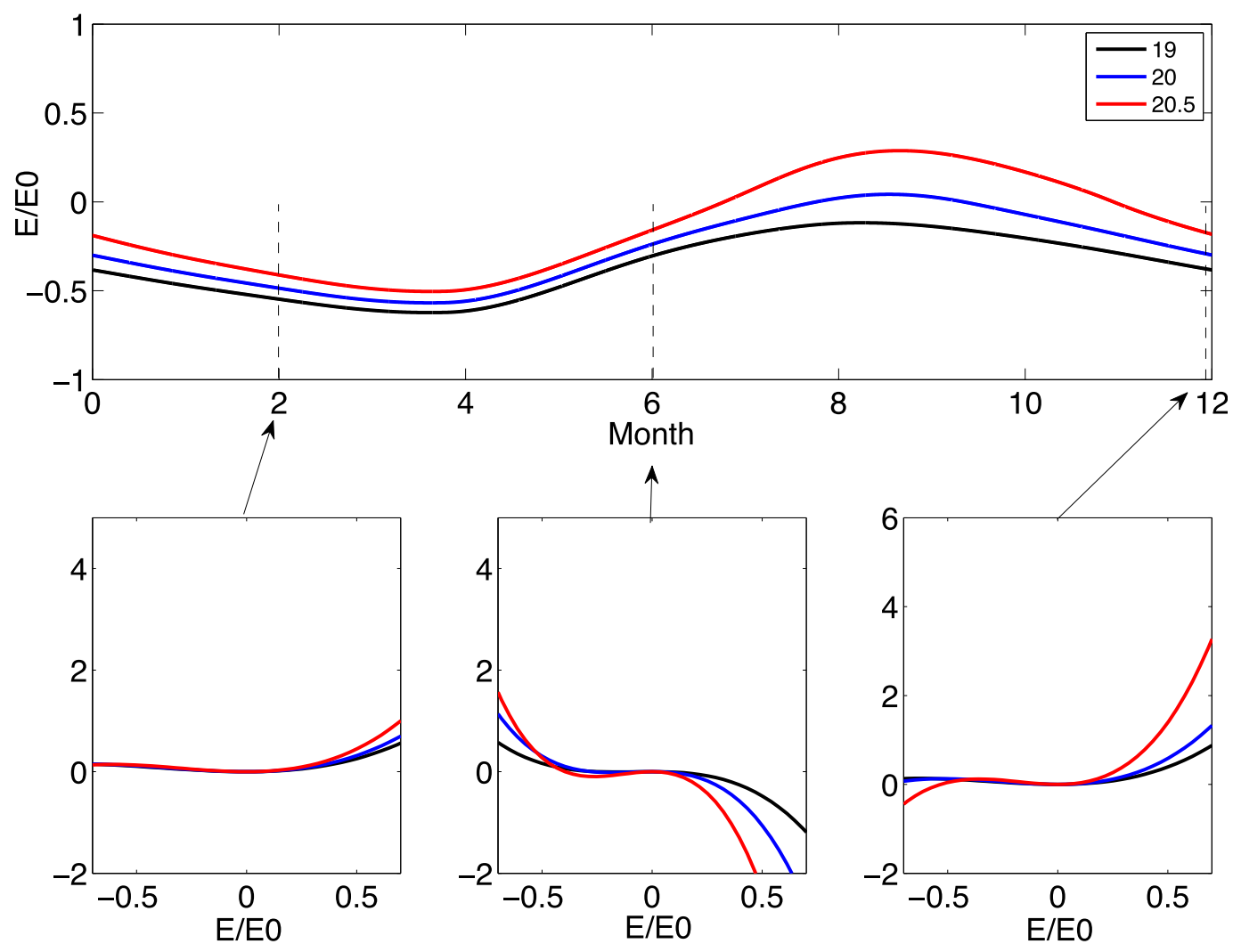

FIG. 5. As in Fig. 3, but for three different values of $\Delta F_{0}: 19.0$ (black), 20.0 (blue), and 20.5 (red). (bottom) The potentials are shown for February, June, and December.

as $\Delta F_{0}$ increases (Eisenman and Wettlaufer 2009). Approaching this transition, when still in the perennial state, the response time scale of a perturbation to the deterministic dynamics is approximately $5 \mathrm{yr}$. However, once the stable seasonally varying state emerges, the response time scale abruptly drops to $2 \mathrm{yr}$ (Moon and Wettlaufer 2011). From the perspective of a stochastic model, this transition is far less clear because noise forcing acts as an additional heat flux source or sink. Intuitively, this implies that the two states can statistically coexist with the same $\Delta F_{0}$, thereby generating a great deal of variability relative to that of states deeply in the perennial ice regime. As $\Delta F_{0}$ further increases, the deterministic system approaches a saddle-node bifurcation from a seasonally varying state to an ice-free state (Eisenman and Wettlaufer 2009). It is important to investigate the variability of these states near the bifurcation point. In this section we study the entire range of $\Delta F_{0}$ spanning these transitions.

\section{1) THE TRANSITION FROM PERENNIAL TO SEASONAL ICE}

The seasonal state appears in the deterministic dynamics as $\Delta F_{0}$ approaches 20.5 from below. Now, we investigate the characteristics of the stochastic solutions near this transition, which is "blurred" in the sense that two stable states coexist at a single $\Delta F_{0}$.

We derive intuition by examining the ice potential $V(E, t)$ near the steady-state solutions, and in Fig. 5 we plot potentials for February, June, and December when $\Delta F_{0}$ is $19.0,20.0$, and 20.5. Because the deterministic steady-state solutions contain very thin sea ice or open ocean during summer, we see enhanced competition between the ice-albedo feedback and the longwave stabilization and hence the asymmetric response of the system to a given perturbation. It is instructive to focus on the potentials for $\Delta F_{0}=20.5$. The potential for June exhibits the ice-albedo feedback through the strong negative slopes when $E / E_{0}>0$. A positive perturbation will grow rapidly away from the steady-state solution; for example, melting leads to more melting resulting from an additional decrease of the ice albedo. By parity of reasoning a negative perturbation leads to more icethe albedo feedback is always positive. However, as we have an energy balance model based on heat conduction and the albedo treatment is based on radiative extinction, the albedo feedback becomes strongly operative once the ice thickness $h \simeq h_{\alpha}=0.5 \mathrm{~m}$. This ice thickness 


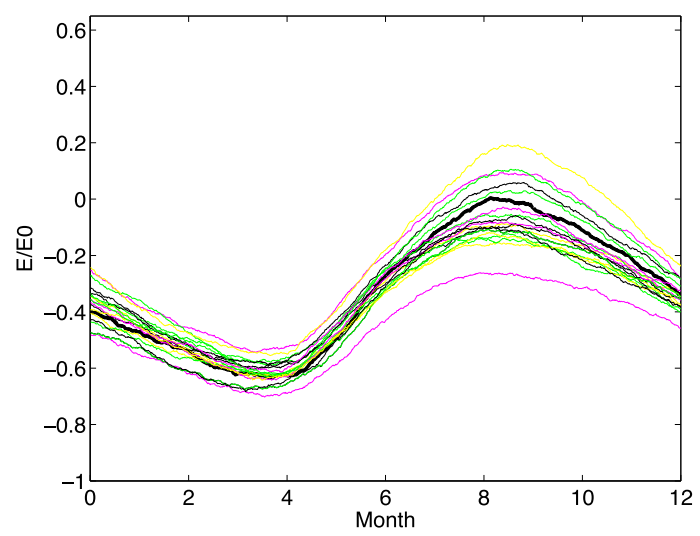

(a)

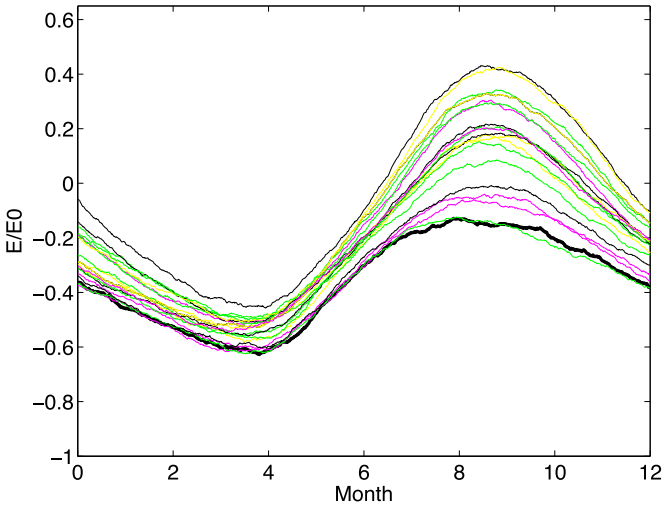

(b)

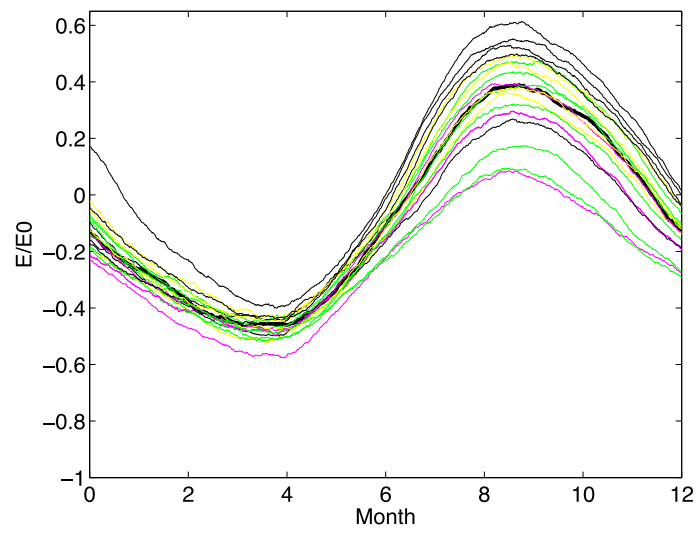

(c)

FIG. 6. As in Fig. 2, but with three different values of $\Delta F_{0}$ : (a) 19.0, (b) 20.0, and (c) 20.5.

enhancement of the asymmetric sensitivity induced by the ice-albedo feedback is fruitfully demonstrated by examining the detailed changes in the ice potentials.

Recall from Fig. 3 that during winter when $\Delta F_{0}$ is such that the system is in the perennial ice state, the potentials are concave and longwave radiative loss strongly stabilizes perturbations in a symmetric manner. However, when $\Delta F_{0}$ increases and the ice is thinner, Fig. 5 shows that during winter the longwave stabilizing response to a perturbation is highly asymmetric. Clearly the slope on the positive side is much larger than that on the negative side and this asymmetry increases with $\Delta F_{0}$.

In Fig. 6 we see stochastic realizations as $\Delta F_{0}$ transitions from perennial to seasonal ice states. (These are discussed in terms of the comparison between additive versus multiplicative noise in more detail in the supplementary material and in section 4.) Although the deterministic steady-state solutions for $\Delta F_{0}=19.0$ and 20.0 are still perennial ice states, the ice is quite thin during the summer and the stochastic realizations tend toward seasonally varying states with ice-free summers. Moreover, while the longwave stabilization is stronger for thinner ice, the ice-albedo feedback dominates, and the asymmetry associated with the latter is stronger than that associated with the former. Recall that as the ice thickness approaches $h_{\alpha}$ the ice albedo changes from that of perennial ice (0.68) to that of open ocean (0.2). Hence, depending on whether the ice thickness is large or small relative to $h_{\alpha}$ the response to a perturbation will be very different. Namely, when $h \approx h_{\alpha}$ the ice is more sensitive to a positive (negative) perturbation, which causes a dramatic increase (decrease) in the albedo. For this reason, near the transition from the perennial to the seasonal ice state, the summer ice thickness approaches $h_{\alpha}$ and a new asymmetry in the stochastic ensemble statistics emerges. Interestingly, we then find that as the system approaches the deterministic transition to seasonal ice, the ice-albedo feedback drives the stochastic solutions toward the seasonal state. However, with only a small increase in $\Delta F_{0}$, the stochastic solutions tend toward the perennial state. This suggests that near the 


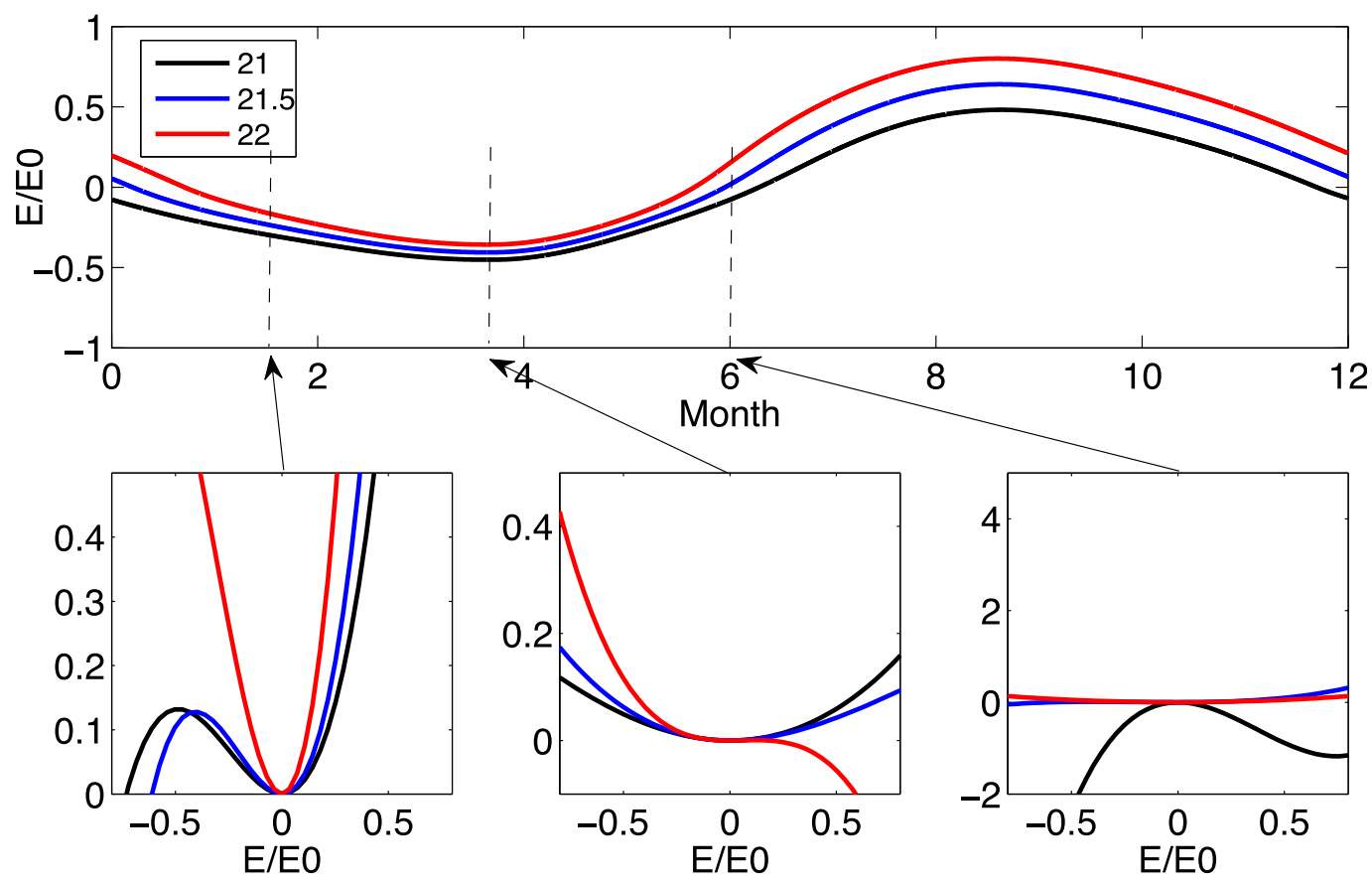

FIG. 7. As in Fig. 3, but for three different values of $\Delta F_{0}: 21.0$ (black), 21.5 (blue), and 22.0 (red). (bottom) The potentials are shown for January, March, and May.

deterministic transition to seasonal ice, the statistical fluctuations in the ice cover can exhibit behavior of both states, and thus the transition itself cannot be explained using concepts based on linear response.

\section{2) APPROACHING THE DETERMINISTIC SADDLE-NODE BIFURCATION}

As $\Delta F_{0}$ increases, the deterministic seasonally varying ice states approach a saddle-node bifurcation to an ice-free state $\left(\Delta F_{0}=23\right)$, which is separated from the perennial state by a hysteresis loop. Here again to examine the stochastic solutions we consider the seasonal cycle of the potentials $V(E, t)$ of the deterministic steady-state solutions for $\Delta F_{0}=21.0,21.5$, and 22.0 (Fig. 7). First, relative to the deterministic steady state for $\Delta F_{0}=20.5$, the dwell time of these solutions in the ice-free state is substantially longer. In particular, note the significant difference in the date at which freeze-up begins between $\Delta F_{0}=20.5$ and 21.0. This highlights the fact that the exposed ocean is an effective heat reservoir and thus acts to prevent the formation of sea ice during the following winter season. The effectiveness of this process depends on the time at which the ice disappears during the summer and hence the time period that the open water is exposed to solar insolation (Moon and Wettlaufer 2012). Indeed, for all three values of $\Delta F_{0}$, sea ice only exists from early January to late May or early June, reflecting the time it takes to remove the stored heat from the mixed layer and bring it to the freezing temperature. Thus, the concave potentials in January represent the onset of heat loss from outgoing longwave radiative flux. As the ice becomes thinner, the curvature near the origin increases. By March, the longwave stabilization weakens and, particularly at $\Delta F_{0}=22.0$, the sea ice-albedo feedback is already operative, which is reflected in the negative slope on the positive side of the potential (red curve). As the summer approaches, in all cases the ice-albedo feedback strengthens and its magnitude increases with $\Delta F_{0}$, as seen through the changes in the slope on the positive side of the potentials. The two main competing physical processes, the longwave stabilization and the ice-albedo feedback, are enhanced substantially during very short time periods. Thus, the sensitivity of the system response to stochastic forcing increases.

The striking behavior that emerges as $\Delta F_{0}$ approaches, but is still less than that for the deterministic saddle-node bifurcation, is seen in the stochastic paths of the seasonal cycle in Fig. 8. For example, some stochastic paths for $\Delta F_{0}=21.5$ shown in Fig. $8 \mathrm{~b}$ exhibit seasonal cycles at the extremes that are both barely seasonal ice states, with small periods of either winter ice or ice-free summers, thereby reflecting the deterministic transition. With only a slight increase in $\Delta F_{0}$ the hysteresis of the deterministic backbone emerges with a 


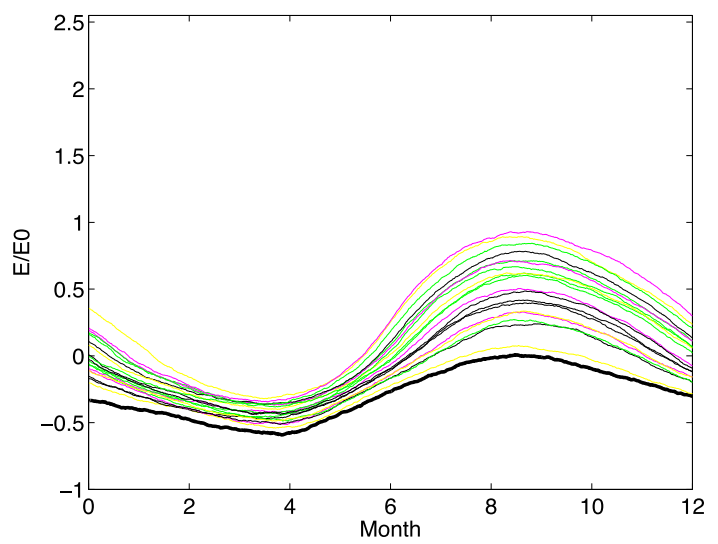

(a)

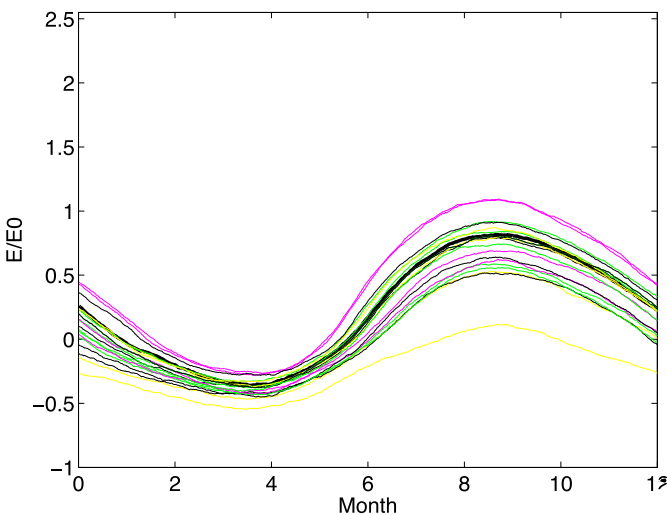

(b)

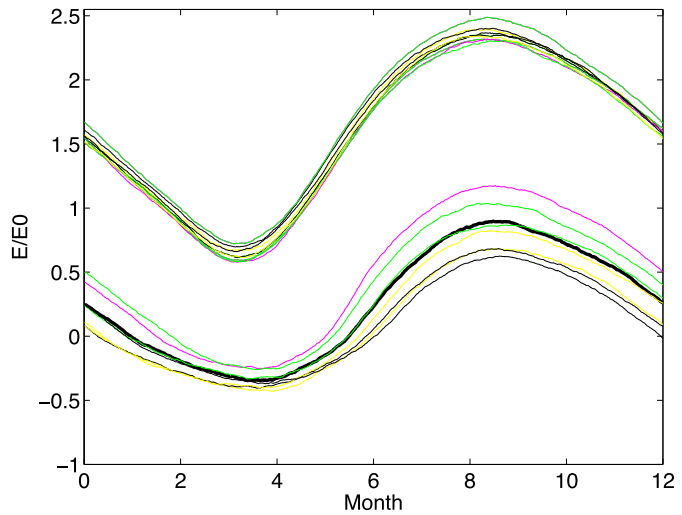

(c)

FIG. 8. As in Fig. 2, but with three different values of $\Delta F_{0}$ : (a) 21.0, (b) 21.5, and (c) 22.0.

two-state stochastic system in which seasonal ice and ice-free states coexist, as seen in Fig. 8c. Importantly, this behavior would manifest itself in a transition with long dwell times in one of these two states and abrupt transitions between them.

\section{Results: Comparing multiplicative and additive noise}

As was done for additive noise, here we analyze the statistical properties of perennial and seasonally varying ice states separately. A physical origin of multiplicative noise is the fluctuations in the surface pressure field, which can be treated as a Gaussian random variable (Thorndike 1982; Agarwal and Wettlaufer 2017). This variability influences, for example, the ice transport from Fram Strait. Clearly, however, there are many other possible sources of noise. As in the case of additive noise, we also use the ability to compare our analytical solutions with the numerical results, in the deterministic regime of perennial ice states where our perturbation theory is valid, as a well-defined test bed of the numerical approach.

\section{a. Perennial ice states}

Stochastic paths are examined for all four cases of additive (CA and SVA) and multiplicative (IM and SM) noise. For an objective comparison among the four cases, we generate the stochastic paths using the same random number at each time step drawn from a normal distribution with zero mean and standard deviation $\sqrt{\Delta t}$. Therefore, the difference between the cases is intrinsic rather than arising from the randomness of the noise forcing. Overall, the stochastic solutions are well approximated by a Gaussian variable with a seasonally evolving standard deviation.

There is no substantial difference between SVA, IM, and SM, but these differ from CA, which has a smaller variability. This is intuitive, because the larger the magnitude of the noise forcing during winter, the more effective it is in generating variability for SVA, IM, and SM than in the case of a seasonally constant noise 
magnitude. According to our perturbation theory (Moon and Wettlaufer 2013), all three cases have the same solution to a first order and are Gaussian variables with standard deviation determined by the combination of the stability of the deterministic seasonal cycle and the noise amplitude. The difference between the multiplicative noise characteristics of IM and SM appears at second order in perturbation theory. The gap between their trajectories represents the intrinsic difference between Itô and Stratonovich calculus. For example, this can be seen as a shift of the stochastic mean due to the cumulative effect of the noise forcing represented explicitly in Stratonovich calculus. In this model the effect is always negative, the origin of which is the deterministic drift term that distinguishes the two calculi (Moon and Wettlaufer 2014), and hence multiplicative noise generates more sea ice.

Clearly, because the solutions are periodic the PDFs change continuously during the year. To demonstrate this change we use the contour diagram shown in Fig. 9, with CA, SVA, and SM in Figs. 9a, 9c, and 9e, respectively, for $\Delta F_{0}=10$. They are quite similar in the sense that the PDFs are broad during summer and become narrow during winter, which is well explained by the two main competing effects of sea ice-albedo feedback and longwave stabilization. The difference between pairs of these PDFs is shown in Figs. 9b,d,f. The difference between SVA and CA shown in Fig. 9b is characterized by the negative region near zero (blue) flanked by the positive regions, which shows that SVA has a wider PDF structure than CA. Note that this effect is particularly strong near the end of April, right before the sea ice-albedo feedback starts to become active. We see that the noise magnitude for SVA is larger than CA during winter when the larger variability resulting from sea ice export is important, after which the sea ice-albedo feedback becomes dominant. The comparison of SM and CA shown in Fig. 9d differs from that between SVA and CA in that the center of the negative region becomes more negative and the positive region on the negative energy side is more pronounced. This qualitative difference becomes more striking in Fig. 9f, which shows that the center of the PDFs for SM become more negative and more negatively skewed.

As $\Delta F_{0}$ increases from 10.0, the competition between the sea ice-albedo feedback and the longwave stabilization is amplified. Slightly thinner sea ice at the end of summer experiences increased longwave stabilization, which is effective throughout the following winter. At the same time, the magnitude of the noise forcing decreases because it is proportional to sea ice thickness, decreasing the overall variability. The imbalance between the longwave stabilization and the sea ice-albedo feedback increases when $\Delta F_{0}=15.0$. However, a further increase in
$\Delta F_{0}$ intensifies the sea ice-albedo feedback thereby increasing the overall variability of the stochastic model.

The contour diagram for $\Delta F_{0}=15.0$ is shown in Fig. 10. The individual PDFs for each case are nearly indistinguishable from each other, so we must examine the differences between them. We see from Figs. 10b,d that the negative region around $E / E_{0}=0$ and the two positive regions flanking it represent the increasing breadth of the PDFs for SVA and SM relative to those for CA. The asymmetry associated with the multiplicative noise effect is shown in Figs. 10d,f, with the increasingly darker red for $E / E_{0}<0$ and the overall negative shift of the PDFs for SM.

Having now examined $\Delta F_{0}=10$ and 15 , we can intuit that a further increase in $\Delta F_{0}$ will enhance the difference between $\mathrm{CA}$ and the other cases. We expect that the seasonal variation of the noise magnitude will generate larger variability and this will couple to the increased influence of the sea ice-albedo feedback during summer. However, as the ice thins, so too will the impact of multiplicative noise, although the relative magnitude of the different contributions to the overall variability are difficult to quantify. For example, as $\Delta F_{0}$ increases the stability of the ice cover weakens, which provides the basis for the enhanced influence of stochastic forcing, but at the same time the magnitude of the noise forcing decreases.

For $\Delta F_{0}=18$ the PDFs of the stochastic solutions start to change dramatically, their spread around the deterministic seasonal cycle showing a strong seasonal dependence, as seen in the contour diagram of Fig. 11. Figures 11a,c,e show that the spread changes dramatically during the year, particularly at the end of a summer, where the standard deviation reaches a maximum. Again, the sea ice-albedo feedback is one of the principal contributors to the stochastic solution structure. The substantial difference between CA and SVA and SM is shown in Figs. 11b,d. The breadth of the PDFs due to the seasonal variation of the magnitude of the noise is exhibited again via the negative region centered around zero, flanked by the two positive regions. The temporal influence of the noise is such that its amplitude saturates in March, but the negative region appears later, between April and May. The multiplicative noise effect shown in Fig. $11 \mathrm{f}$ is somewhat diminished relative to $\Delta F_{0}=10$. In particular, the positive regions (red) on the negative (lower) side show that the negative tail of the PDFs is weaker than in the case with $\Delta F_{0}=10.0$.

In summary, as $\Delta F_{0}$ increases from 10.0 to 19.0 , the deviation of the stochastic mean from the deterministic seasonal cycle changes from negative to positive for all the four cases, the difference between CA and SVA becoming larger with $\Delta F_{0}$. The noise forcing-induced by the variability of sea ice export provides two 


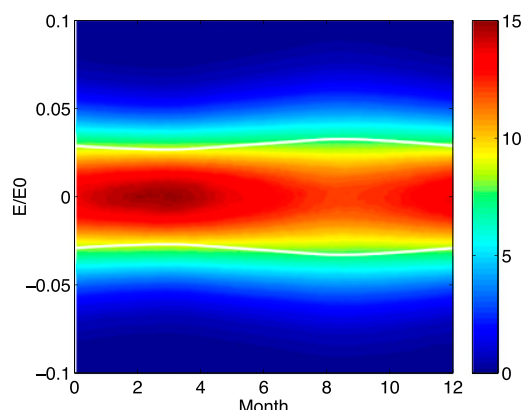

(a)

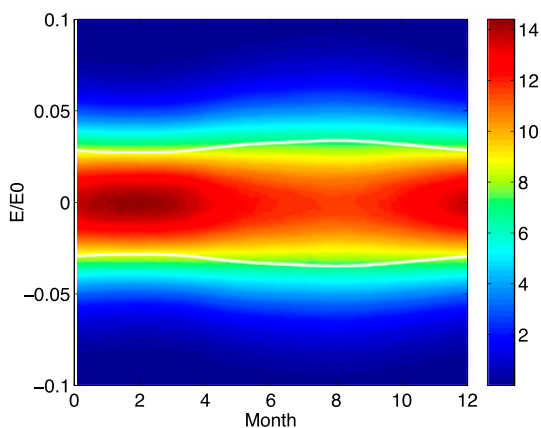

(c)

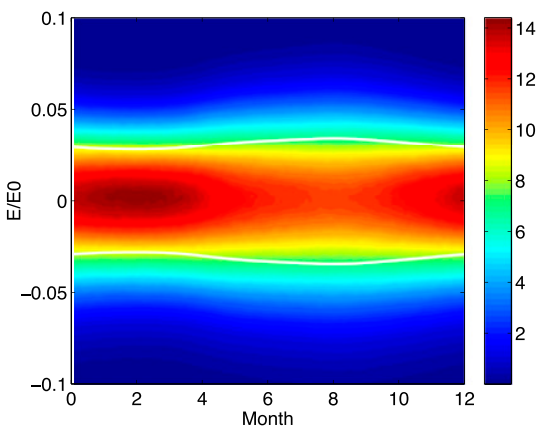

(e)

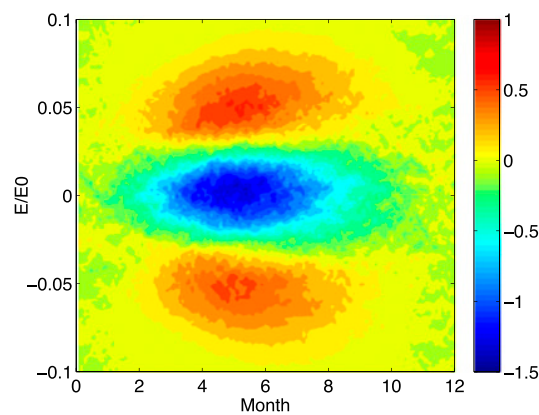

(b)

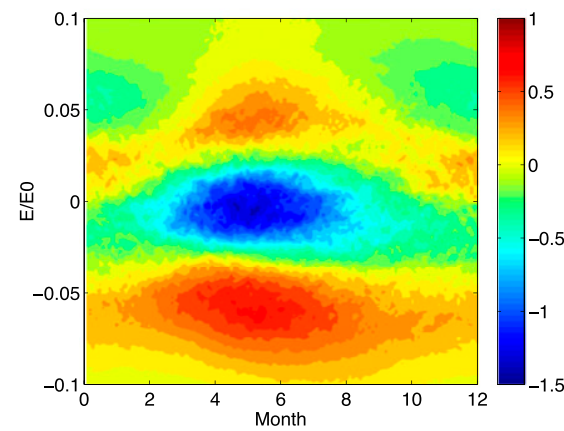

(d)

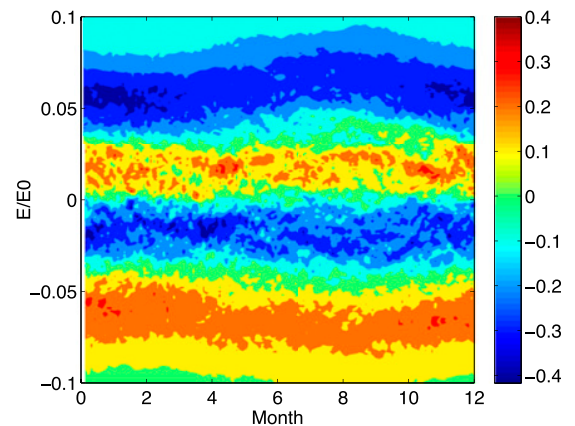

(f)

FIG. 9. Seasonal evolution of PDFs for (a) CA, (c) SVA, and (e) SM and the difference of the PDFs (b) between SVA and CA, (d) between SM and CA, and (f) between SM and SVA are shown when $\Delta F_{0}=10.0$, in the perennial state of the deterministic system. The $x$ axis is the month of the year from January to December, and the $y$ axis the rescaled sea ice energy as in the previous figures. The probability density is shown by the color scheme, where red represents larger values. The deterministic seasonal cycle is indicated by $E / E_{0}=0$, and the two white lines centered around $E / E_{0}=0$ indicate the standard deviation of the stochastic solutions.

important factors controlling the statistics of the stochastic solutions: 1) the seasonal change of the noise magnitude and 2) the effect of multiplicative noise. The larger the magnitude of the noise near the end of winter the more effective it is in generating increased variability of sea ice energy, and this becomes more important as $\Delta F_{0}$ increases. The effect of multiplicative noise, which always reduces the stochastic mean and the skewness, is stronger for lower values of $\Delta F_{0}$ because the noise magnitude is proportional to the sea ice thickness. We end this section by noting that the approximate analytical solutions match well with the numerical solutions, suggesting that further research regarding the perennial ice states may be fruitfully explained using approximate methods (Moon and Wettlaufer 2013).

\section{b. Seasonally varying states}

As $\Delta F_{0}$ increases, the deterministic dynamics predicts a reversible transition from perennial to seasonal ice, 


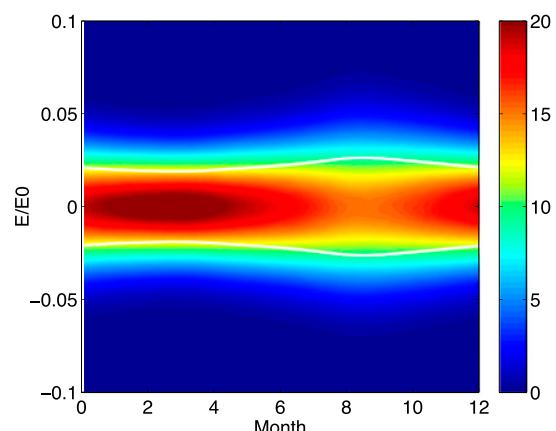

(a)

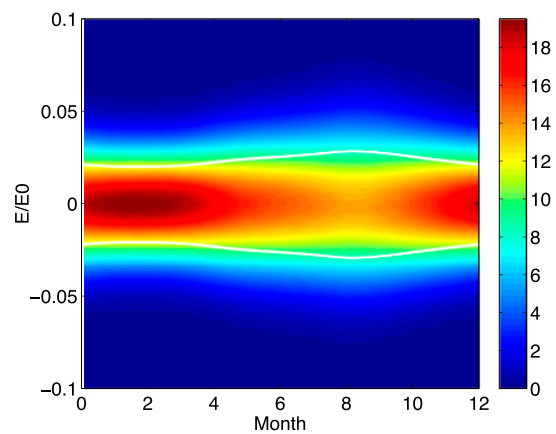

(c)

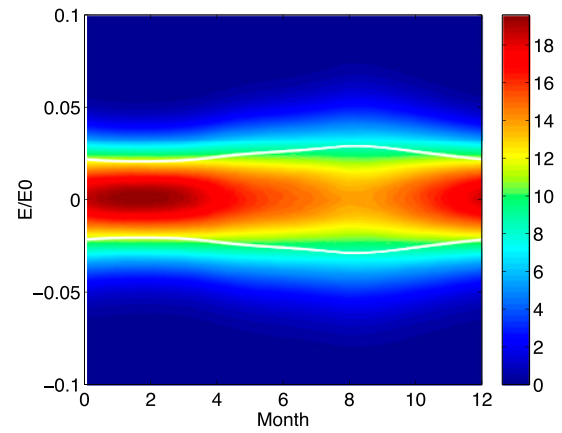

(e)

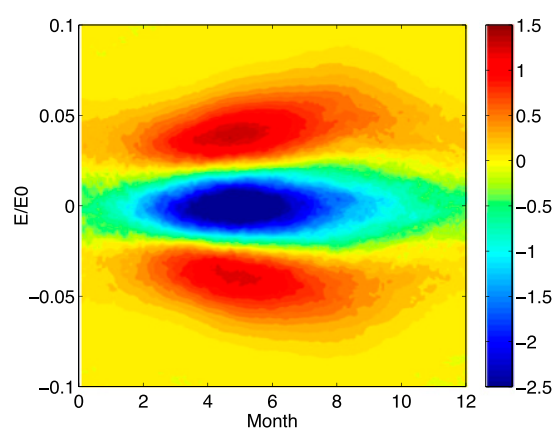

(b)

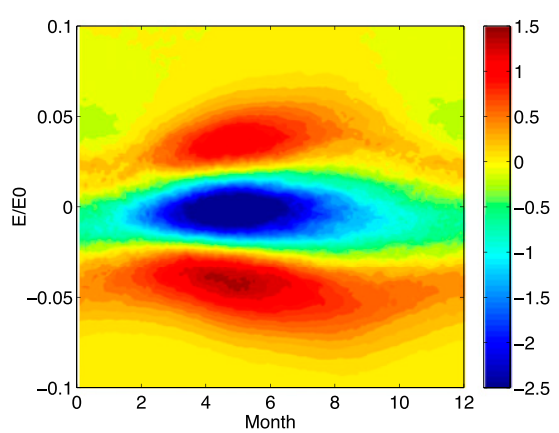

(d)

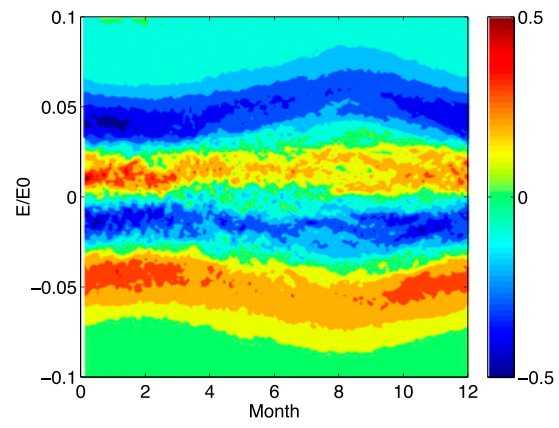

(f)

FIG. 10. As in Fig. 9, except that $\Delta F_{0}=15.0$.

where sea ice vanishes during summer and grows back during winter. It is notable that on the annual time scale the observed ice extent is a white noise signal (Agarwal et al. 2012), and we find here that the seasonal states undergo dramatic fluctuations during the year. Thin ice exposed to strong shortwave radiative flux during early summer melts quickly as a result of the sea ice-albedo feedback. As winter approaches, thin ice forms from the open ocean and then grows rapidly because of the strength of the longwave radiative heat loss. Regardless of the structure of the noise, its effect is to generate large variability around the deterministic seasonal cycle. Unfortunately, as mentioned above and previously (Moon and Wettlaufer 2013), analytical solutions are not yet in hand for this regime. Nonetheless, the logic found in studying the perennial ice state acts as a framework for understanding stochastic solutions in the seasonal case.

The continuous evolution of the PDFs over the year for all of the noise cases when $\Delta F_{0}=20.0$ is shown in Fig. 12. Even though the overall magnitude of the noise is smaller than that for the perennial sea ice states, the stochastic variability is even larger, which reflects the reduced stability of the system. In the constant additive noise case, we found that the PDFs at the same $\Delta F_{0}$ have positive tails due to the increased seasonal influence of the sea ice-albedo feedback.

A key common characteristic of the PDFs is the distinct difference between summer and winter. The standard deviations (the two white lines) exhibit a dramatic change from winter to summer. Accordingly, the shape of the 


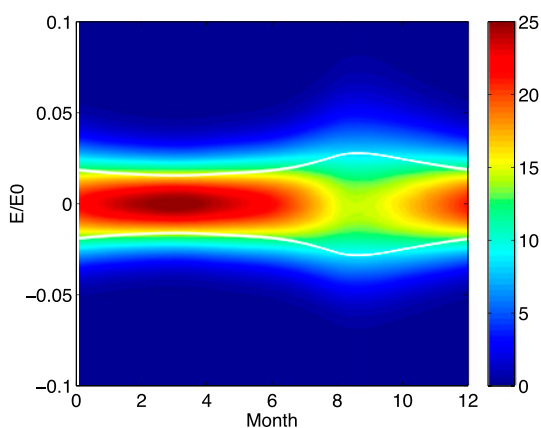

(a)

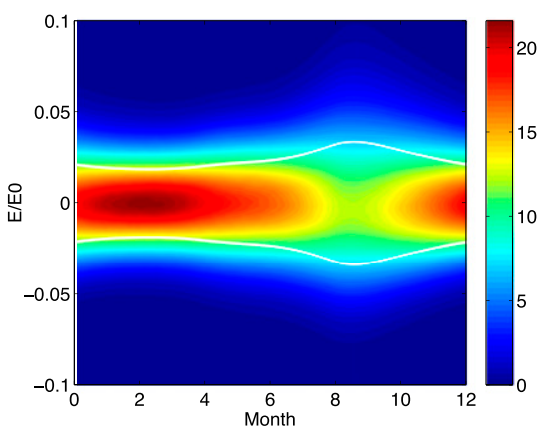

(c)

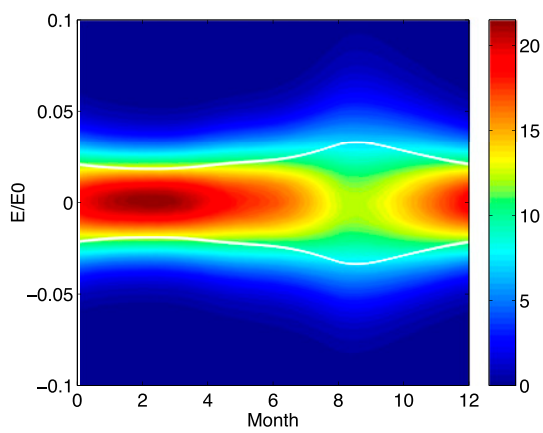

(e)

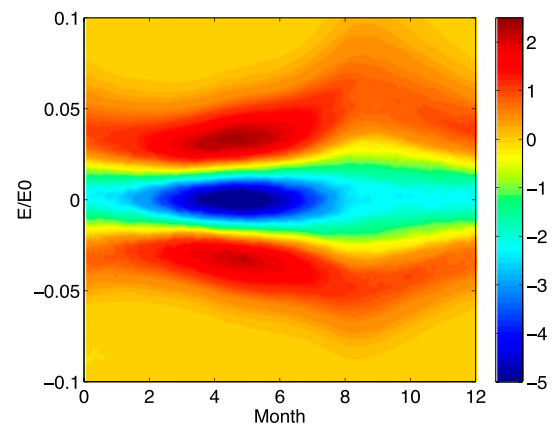

(b)

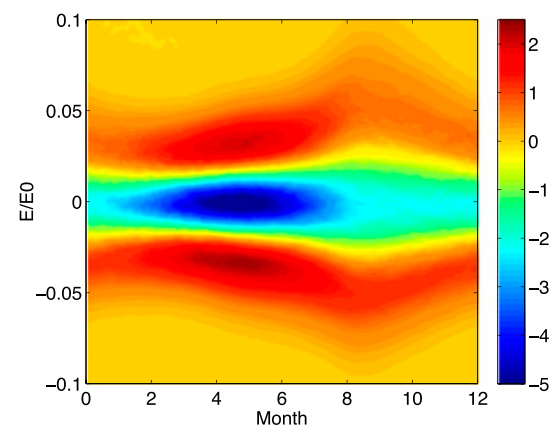

(d)

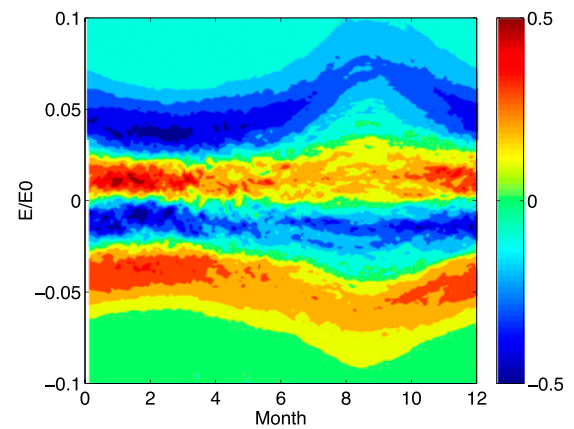

$(\mathrm{f})$

FIG. 11. As in Fig. 9, except that $\Delta F_{0}=18.0$

PDFs also changes from sharply peaked to broad and the positive tails extend further in the positive sense during summer. These general characteristics are seen in CA, SVA, and SM. The difference between SVA and CA, shown in Fig. 12b, or between SM and CA, shown in Fig. 12d, is qualitatively similar to the warmer (larger $\left.\Delta F_{0}\right)$ perennial ice states that exhibited continuous broadening. The difference between SVA and SM, shown in Fig. 12f, is also similar to these previous cases, exhibiting a negative shift of the PDFs resulting from the drift term.

As $\Delta F_{0}$ increases slightly above 20.0 we find large differences between the solutions. First, the deterministic seasonal cycle changes rapidly with an increase in $\Delta F_{0}$ in this regime, for example, the open-ocean state persists much longer. Moreover, the sea ice-albedo feedback becomes more sensitive to negative energy perturbations, which means that stochastic forcing generates more sea ice. Because the ice is thinner, the noise amplitude is smaller and the variability for all four cases decreases.

All of the PDFs for $\Delta F_{0}=20.5$ have negative tails (Fig. 13), which is explained by the increased sensitivity of the albedo feedback to negative energy perturbations; the growth rate for a negative perturbation during summer is larger than that for a positive one. The qualitative consistency with the constant noise case is due to the decreased magnitude of the overall noise forcing during the year. The seasonal variation of the noise forcing and the effect of multiplicative noise do not make a significant difference. Thus, as expected, the PDFs during summer have broader negative tails. 


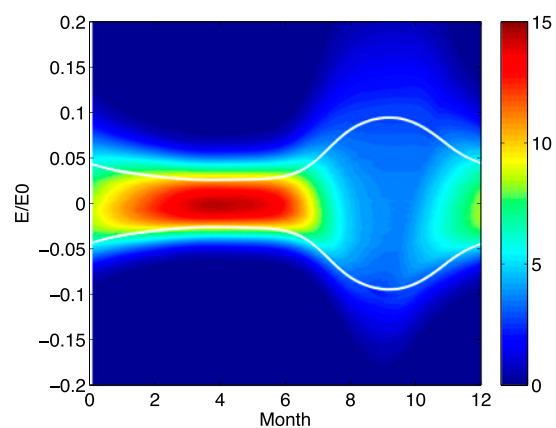

(a)

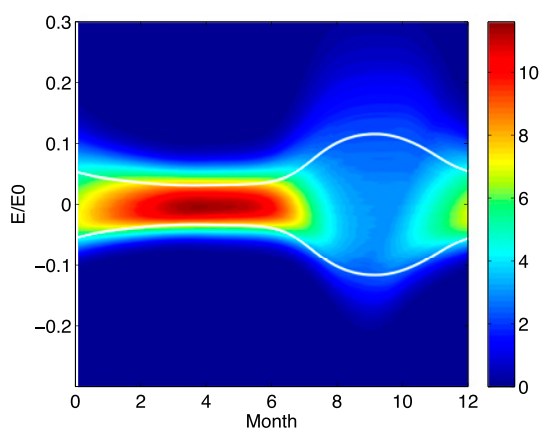

(c)

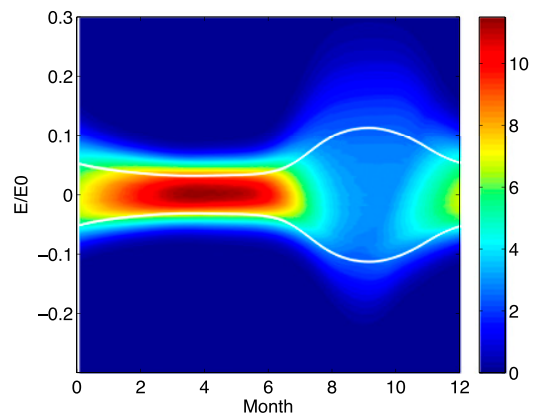

(e)

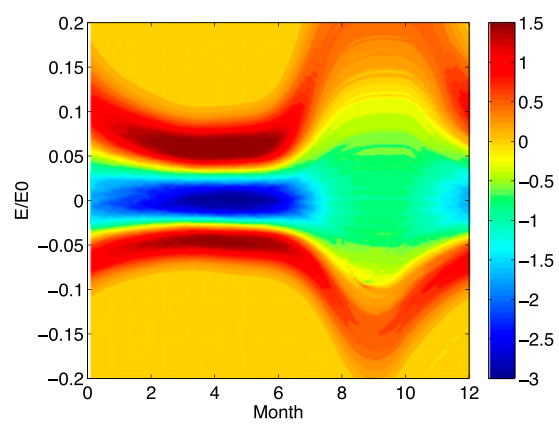

(b)

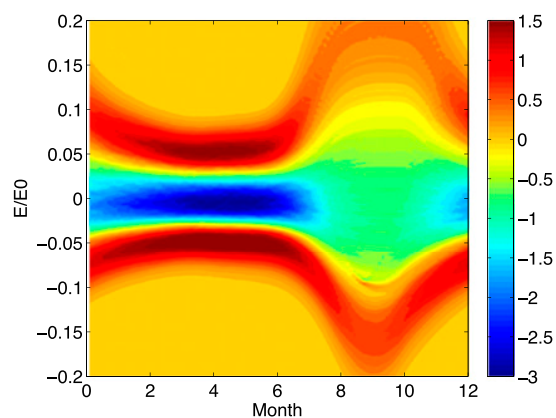

(d)

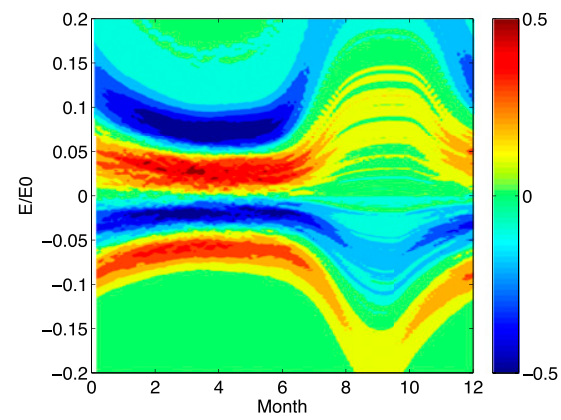

(f)

FIG. 12. As in Fig. 9, except that $\Delta F_{0}=20.0$.

One of the most important characteristics of the CA, SVA, and SM cases is the clear contrast between summer and winter, as seen in Figs. 13a,c,e, the origin of which is the dramatic change in the seasonal stability of the ice cover. The difference between CA and SVA shown in Fig. 13b is similar to the previous cases, with seasonal broadening and contraction. The PDFs for SM become more negative than those for SVA, shown as an increase in the red intensity straddling $E / E_{0}=0$ in Figs. 13d,f.

The statistical moments are sensitive to small changes in $\Delta F_{0}$ in the seasonal state. First, the standard deviation is slightly smaller than that at the lower $\Delta F_{0}$, which is due to the decreased noise amplitude associated with the overall decay of the ice cover. Contrary to the sharp decrease after the maximum, the standard deviation decreases slowly after reaching the maximum and then shows a sharp decline in approximately November. Recall that at this time there is open ocean, which has a large sensible heat and must be cooled before freezing can begin. After the ice forms, the strong longwave stabilization plays an important role in suppressing fluctuations. The deviation of the stochastic mean from the deterministic seasonal cycle is largely negative for all of the four cases. After the local maximum in June, a significant negative shift appears, which represents the sea ice-albedo feedback being more sensitive to negative perturbations. Finally, near the transition from the open ocean to thin sea ice, there exists another local maximum. The first peak is associated with the sea icealbedo feedback in early summer, and the second peak is the emergence of thin sea ice from open ocean. When thin sea ice is generated, a perturbation can be negative 


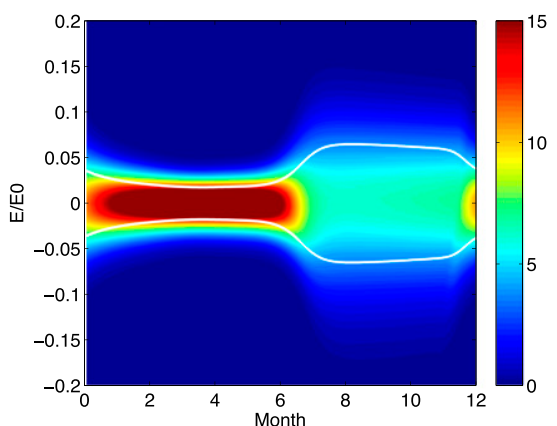

(a)

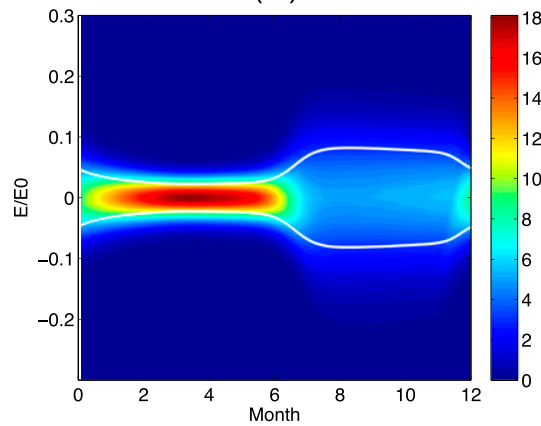

(c)

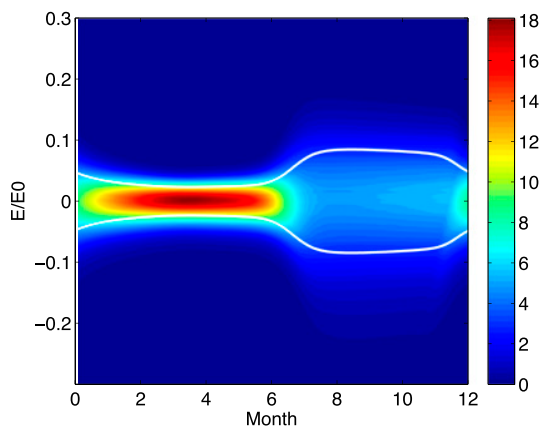

Month

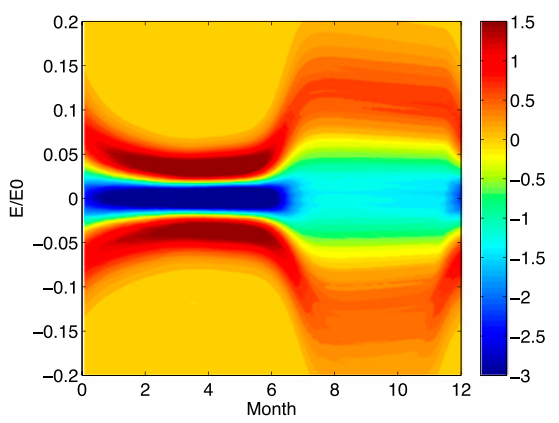

(b)

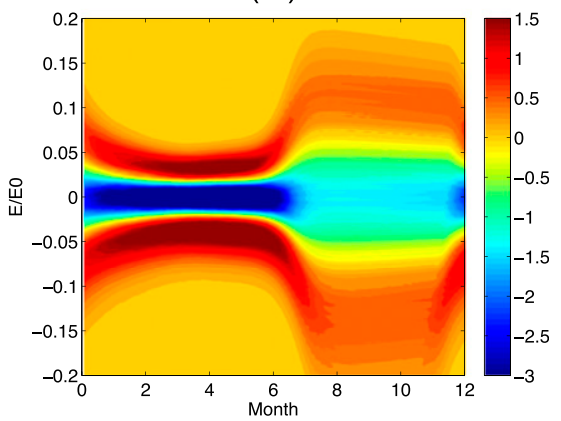

(d)

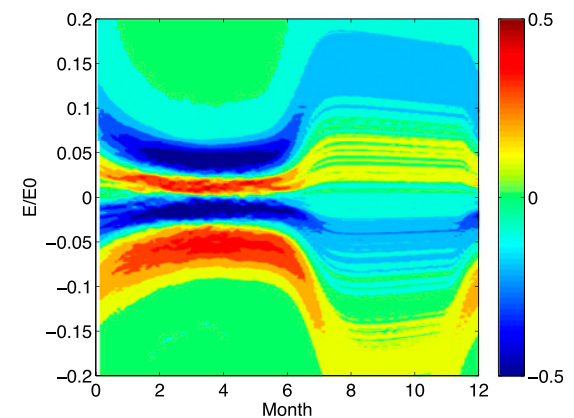

$(\mathrm{f})$

FIG. 13. As in Fig. 9, except that $\Delta F_{0}=20.5$.

or positive. A positive perturbation leads to temporary melting of thin ice. The open ocean has high heat capacity and thus stores substantial sensible heat, which always acts to delay the formation of thin sea ice. Therefore, the system has positive asymmetry during the early stages of thin ice generation. After the ice is sufficiently thick, the strong longwave stabilization begins to control the stochastic solutions.

We summarize the statistics of the stochastic solutions in the seasonally varying state as follows. The standard deviation for SVA is larger than that for CA over the entire range of $\Delta F_{0}$, which was also seen for the perennial ice regime. The standard deviation is almost the same for SVA and SM, but a visible difference emerges near the deterministic saddle-node bifurcation. In the deviation of the stochastic mean from the deterministic seasonal cycle and the skewness, it is important to focus on the role of the sea ice-albedo feedback near the deterministic transition from the perennial to the seasonally varying ice state. For $\Delta F_{0} \approx 19.0$ (below the transition), both quantities are positive, which is associated with the nature of the ice-albedo feedback and is, as expected, enhanced for the SVA case. For the SM case, the negative multiplicative noise effect ensures lower values than for the SVA case. As discussed above, a slight increase in $\Delta F_{0}$ leads to a substantially different situation, as is evident in the negative deviation of the stochastic mean from the deterministic solution and the negative skewness. The increased sensitivity of the ice-albedo feedback to a negative perturbation dominates the statistics immediately after the emergence of seasonally varying states. After passing through the 
transition, a sharp increase in the deviation of the stochastic mean from the deterministic solution and the skewness occurs until the deterministic saddle-node bifurcation to an ice-free state is approached. Distinctions with the cases at lower $\Delta F_{0}$ include the skewness and the noise magnitude for CA being larger than that for SVA, where the noise magnitude is proportional to the ice thickness. Additionally, the skewness for SM is larger than that for SVA and the skewness for the winter is larger than that for the summer. The summer value is taken at the end of August when the open ocean is stable relative to thin sea ice. At the end of March thin sea ice remains, which generates substantial sensitivity to perturbations.

\section{Conclusions}

Using both analytical and numerical methods, we have studied the dependence of the solutions of a stochastic sea ice model on the external heat flux $\Delta F_{0}$, which models greenhouse gas forcing, for both additive and multiplicative noise. Additive noise does not depend on the state of the system itself and is thus qualitatively and quantitatively distinct from multiplicative noise, which does depend on the state of the system. Here, in the latter case we considered the variability of atmospheric forcing driving a variation of sea ice export as a key source of multiplicative noise, and hence the noise forcing is linearly proportional to the sea ice thickness (or energy). The ensemble statistics of the system depend upon the stability and asymmetry of the underlying deterministic solutions and the magnitude of the noise forcing. The stability and the asymmetry are principally determined by two main processes; the icealbedo feedback and the longwave stabilization, which act asynchronously.

We divided the analysis into the three regimes of $\Delta F_{0}$ associated with the steady-state solutions of the deterministic system: perennial and seasonal ice and ice-free states as found by Eisenman and Wettlaufer (2009). The deterministic perennial and seasonal states are separated by a reversible transition, and the seasonal and ice-free states are delineated by a saddlenode bifurcation. By introducing the concept and an "ice potential," which describes the thermodynamic restoring forces in the system in a manner akin to a time-dependent Ornstein-Uhlenbeck process, we provide a relatively simple framework for interpreting the solutions.

Because the underlying deterministic model is nonautonomous, so too is the stochastic model. When the noise magnitude is small, and $\Delta F_{0}$ is such that the deterministic solutions are in the perennial state, we can compare numerical simulations with perturbative solutions derived previously (Moon and Wettlaufer 2013). This allows us to distinguish between the core nonlinear effects of the deterministic backbone of the model from those associated with noise forcing at each order in the perturbative framework. We find a "memory effect" whereby the intrinsic nonlinearity, asymmetry, and stability characteristics of the interaction between the deterministic backbone and the noise allow fluctuations in ice energy from the early spring to accumulate and manifest themselves in the late summer.

We constructed and examined four variants of this noise structure for a detailed comparison with the perturbative solution in the deterministic regime of stable perennial ice states. The most general form of multiplicative noise forcing is $\sigma \mathcal{R}[-E(t)] \xi(t)$, where $|\sigma| \ll 1$ is the magnitude of the noise, $E(t)$ is the sea ice energy, and $\xi(t)$ is white noise. Two cases were considered here, depending on the nature of the stochastic calculus: Itô calculus (IM), which preserves the Martingale property, and Stratonovich calculus (SM), where the $\mathrm{M}$ denotes multiplicative. Because analysis of the properties of data alone is insufficient to determine which of the stochastic calculi is most appropriate for the task at hand, in the case of multiplicative noise we compare simulations from both Itô and Stratonovich calculi. The core reason for this is insufficient information regarding the difference in time scale between noise forcing, inertia, and/or feedbacks in the system, as is discussed in detail in section $2 \mathrm{c}$ above. The seasonally varying noise (SVA) case, with noise amplitude $\sigma \mathcal{R}\left(-E_{S}\right)$, where $E_{S}(t)$ is the deterministic steady-state solution, examines the role of the seasonal change of the noise amplitude. The constant additive noise (CA) case uses the seasonal average of $E_{S}(t)$ and thus has noise amplitude $\sigma \overline{\mathcal{R}\left[-E_{S}(t)\right]}$, where the overbar denotes the seasonal time average.

In the perennial ice regime the difference between CA and SVA reveals the role of the seasonal variation of the noise amplitude and is detectable at a first order in perturbation theory, where the approximate solution is a Gaussian variable. As expected from the perturbation theory, the SM and IM cases exhibit no distinct difference with SVA at a first order. Rather, their differences are found at second order where non-Gaussian characteristics were predicted theoretically. Specifically, the difference between SVA and IM is seen in the skewness, because of the role of the effect of the multiplicative noise. The difference between IM and SM is due to the shift of the mean associated with the drift term in Stratonovich calculus.

Even though the magnitude of the noise for the SVA case is larger (smaller) than that for CA during winter (summer), the seasonal standard deviation is larger. The 
overall behavior represents the confluence of the seasonal memory effect with the variation of the noise magnitude. As the external heat flux $\Delta F_{0}$ increases in this regime, the standard deviation decreases because of the decline of the noise magnitude with the decline in ice thickness, but increases again as $\Delta F_{0}$ increases further, because of the weakened stability associated with the ice-albedo feedback. This change in the standard deviation with increasing $\Delta F_{0}$ explains the first-order solutions for all of the cases.

For small $\Delta F_{0}$ the deviation of the stochastic mean from the deterministic seasonal cycle is negative for all of the cases, with SM having a larger deviation as a result of the nature of the mean shift induced by multiplicative noise. As $\Delta F_{0}$ increases further, the stochastic mean becomes larger than the deterministic seasonal cycle due to the icealbedo feedback. The difference between SVA and CA is particularly distinct, showing that the larger magnitude of the noise at the end of winter continues to impact the fluctuations of the sea ice energy during summer, which is the memory effect. The skewness behaves similarly to the deviation of the stochastic mean from the deterministic solution. The negative skewness for smaller values of $\Delta F_{0}$ increases sharply and becomes positive as $\Delta F_{0}$ increases. The effect of multiplicative noise in the SM and IM cases drives the sea ice energy toward negative values such that the skewness for these cases is smaller than that for SVA. The numerical results match the perturbation solutions nearly exactly, confirming the validity of the theoretical analysis in the perennial ice regime.

The seasonally varying states are clearly qualitatively and quantitatively different than the perennial states. For example, the difference between SVA and CA is larger than in the perennial ice regime. Thus, quantitative estimation of sea ice variability in the seasonal state depends sensitively upon the detailed nature of the seasonality of the noise magnitude. The controlling factor in the variability is the increased sensitivity of the ice-albedo feedback to negative energy (positive thickness) fluctuations near the transition from the perennial to the seasonally varying regime. This signed sensitivity leads to both the deviation of the stochastic mean from the deterministic solution and the skewness having local minima near $\Delta F_{0}=20.5$, which is most pronounced in the SVA case. These statistics pass through a smaller minimum in the SM and IM cases because of the nature of the multiplicative noise. Finally, all of the statistical moments increase sharply as $\Delta F_{0}$ approaches the deterministic saddle-node bifurcation.

The central complexities of the evolution of the stochastic solutions as $\Delta F_{0}$ increases through the perennial and seasonally varying regimes of Arctic sea ice are best embodied in the evolution of the PDFs shown in
Figs. 9-12. Regardless of the regime, as $\Delta F_{0}$ increases the seasonality of the variability increases but is maximal in the seasonal state. The structure of other moments reveals the distinctions between additive and multiplicative noise, which becomes acutely important as the stability of the deterministic seasonal cycle weakens. The asymmetry associated with the ice-albedo feedback response manifests itself in qualitatively unique ways when fluctuations are (not) tied to the ice energy and/or thickness in multiplicative (additive) noise. There are a number of processes in which multiplicative noise is tied to observational reality, but as a general feature (independent of its origin) in this sort of a model it possesses some compelling features. First, it captures leading-order growth or decay of fluctuations, which we expect from general considerations of simple Langevin equations. Second, in the case we considered here, as the ice cover is reduced then the fluctuations are less effective in impacting the state of the system, and this is clearly seen in the variability of the seasonal cycle and the nature of the memory effect. It is thus of interest to systematically and explicitly incorporate stochastic effects in more complex models of sea ice, as is done in atmospheric models (Dawson and Palmer 2015). To this end, the framework provided here may be of use.

Acknowledgments. WM acknowledges a NASA Graduate Research Fellowship and a Herchel-Smith postdoctoral fellowship. JSW acknowledges Swedish Research Council Grant 638-2013-9243, a Royal Society Wolfson Research Merit Award, and NASA Grant NNH13ZDA001N-CRYO for support. Much of this work was completed at the 2015 Geophysical Fluid Dynamics Summer Study Program "Stochastic Processes in Atmospheric \& Oceanic Dynamics" at the Woods Hole Oceanographic Institution, which is supported by the National Science Foundation and the Office of Naval Research. We thank many of the staff for comments and criticisms.

\section{REFERENCES}

Agarwal, S., and J. S. Wettlaufer, 2017: The statistical properties of sea ice velocity fields. J. Climate, doi:10.1175/ JCLI-D-16-0653.1, in press.

_- W. Moon, and J. S. Wettlaufer, 2012: Trends, noise and reentrant long-term persistence in Arctic sea ice. Proc. Roy. Soc. London, 468A, 2416-2432, doi:10.1098/rspa.2011.0728.

Benzi, R., A. Sutera, and A. Vulpiani, 1981: The mechanism of stochastic resonance. J. Phys., 14A, L453-L457, doi:10.1088/ 0305-4470/14/11/006.

Budyko, M. I., 1969: Effect of solar radiation variation on climate of Earth. Tellus, 21A, 611-619, doi:10.3402/tellusa.v21i5.10109.

Dawson, A., and T. N. Palmer, 2015: Simulating weather regimes: Impact of model resolution and stochastic parameterization. Climate Dyn., 44, 2177-2193, doi:10.1007/s00382-014-2238-x. 
Dijkstra, H. A., 2013: Nonlinear Climate Dynamics. Cambridge University Press, $367 \mathrm{pp}$.

Doering, C. R., 2016: Mathematical foundations of stochastic processes. 2015 Program of Study: Stochastic Processes in Atmospheric and Oceanic Dynamics, J. S. Wettlaufer and O. Bühler, Eds., Woods Hole Oceanographic Institution Tech. Rep. WHOI-2016-05, 1-40, doi:10.1575/1912/8601.

Eisenman, I., and J. S. Wettlaufer, 2009: Nonlinear threshold behavior during the loss of Arctic sea ice. Proc. Natl. Acad. Sci. USA, 106, 28-32, doi:10.1073/pnas.0806887106.

—, T. Schneider, D. S. Battisti, and C. M. Bitz, 2011: Consistent changes in the sea ice seasonal cycle in response to global warming. J. Climate, 24, 5325-5335, doi:10.1175/2011JCLI4051.1.

Hasselmann, K., 1976: Stochastic climate models part I. Theory. Tellus, 28A, 473-485, doi:10.3402/tellusa.v28i6.11316.

Held, I. M., and M. J. Suarez, 1974: Simple albedo feedback models of the icecaps. Tellus, 26, 613-629, doi:10.3402/tellusa.v26i6.9870.

Kloeden, P. E., and E. Platen, 1992: Numerical Solution of Stochastic Differential Equations. Stochastic Modelling and Applied Probability, Vol. 23, Springer, 636 pp.

Kupferman, R., G. A. Pavliotis, and A. M. Stuart, 2004: Itô versus Stratonovich white-noise limits for systems with inertia and colored multiplicative noise. Phys. Rev., 70E, 036120, doi:10.1103/PhysRevE.70.036120.

Kwok, R., and N. Untersteiner, 2011: The thinning of Arctic sea ice. Phys. Today, 64, 36-41, doi:10.1063/1.3580491.

_ - G. F. Cunningham, and S. S. Pang, 2004: Fram Strait sea ice outflow. J. Geophys. Res., 109, C01009, doi:10.1029/ 2003JC001785.

Lemke, P., 1986: Stochastic description of atmosphere-sea iceocean interaction. The Geophysics of Sea Ice, N. Untersteiner, Ed., NATO ASI Series, Vol. 146, Springer, 785-823.

Lindzen, R. S., and B. Farrell, 1977: Some realistic modifications of simple climate models. J. Atmos. Sci., 34, 1487-1501, doi:10.1175/1520-0469(1977)034<1487:SRMOSC > 2.0.CO;2.

Maykut, G. A., and N. Untersteiner, 1971: Some results from a time-dependent thermodynamic model of sea ice. J. Geophys. Res., 76, 1550-1575, doi:10.1029/JC076i006p01550.

Meier, W. N., and Coauthors, 2014: Arctic sea ice in transformation: A review of recent observed changes and impacts on biology and human activity. Rev. Geophys., 52, 185-217, doi:10.1002/2013RG000431.

Moon, W., and J. S. Wettlaufer, 2011: A low-order theory of Arctic sea ice stability. Europhys. Lett., 96, 39001, doi:10.1209/ 0295-5075/96/39001.

— and - 2012: On the existence of stable seasonally varying Arctic sea ice in simple models. J. Geophys. Res., 117, C07007, doi:10.1029/2012JC008006.

—, and -2013 : A stochastic perturbation theory for nonautonomous systems. J. Math. Phys., 54, 123303, doi:10.1063/ 1.4848776 .

— and - 2014: On the interpretation of Stratonovich calculus. New J. Phys., 16, 055017, doi:10.1088/1367-2630/16/5/055017.
Nicolis, C., and G. Nicolis, 1981: Stochastic aspects of climatic transitions-additive fluctuations. Tellus, 33A, 225-234, doi:10.3402/tellusa.v33i3.10710.

North, G. R., 1975: Analytical solution to a simple climate model with diffusive heat transport. J. Atmos. Sci., 32, 1301-1307, doi:10.1175/1520-0469(1975)032<1301:ASTASC > 2.0.CO;2.

— chastic climate model. J. Atmos. Sci., 38, 504-513, doi:10.1175/ 1520-0469(1981)038<0504:PIASSC $>2.0$. CO;2.

Pesce, G., A. McDaniel, S. Hottovy, J. Wehr, and G. Volpe, 2013: Stratonovich-to-Itô transition in noisy systems with multiplicative feedback. Nat. Commun., 4, 2733, doi:10.1038/ ncomms 3733 .

Risken, H., 1984: The Fokker-Planck Equation: Methods of Solution and Applications. Springer Series in Synergetics, Vol. 18, Springer, $454 \mathrm{pp}$.

Rose, B. E. J., and J. Marshall, 2009: Ocean heat transport, sea ice, and multiple climate states: Insights from energy balance models. J. Atmos. Sci., 66, 2828-2843, doi:10.1175/ 2009JAS3039.1.

Saltzman, B., 2002: Dynamical Paleoclimatology: Generalized Theory of Global Climate Change. International Geophysics Series, Vol. 80, Academic Press, 354 pp.

Sellers, W., 1969: A global climatic model based on the energy balance of the earth-atmosphere system. J. Appl. Meteor., 8, 392-400, doi:10.1175/1520-0450(1969)008<0392:AGCMBO > 2.0.CO;2.

Shreve, S. E., P. Chalasani, and S. Jha, 2004: Stochastic Calculus for Finance. Vol I, The Binomial Asset Pricing Model, Springer, $187 \mathrm{pp}$.

Stefan, J., 1889: Über einige probleme der theorie der wälarmeleitung. Sitzungsber. Dtsch. Akad. Wiss. Berlin, Math.-Naturwiss. Kl., 98, 473-484.

Thorndike, A. S., 1982: Statistical properties of the atmospheric pressure field over the Arctic Ocean. J. Atmos. Sci., 39, 2229-2238, doi:10.1175/1520-0469(1982)039<2229:SPOTAP >2.0.CO;2.

_ 1992: A toy model linking atmospheric thermal radiation and sea ice growth. J. Geophys. Res., 97, 9401-9410, doi:10.1029/ 92JC00695.

Tocino, A., and J. Vigo-Aguiar, 2002: Weak second order conditions for stochastic Runge-Kutta methods. SIAM J. Sci. Comput., 24, 507-523, doi:10.1137/S1064827501387814.

Tredicce, J. R., G. L. Lippi, P. Mandel, B. Charasse, A. Chevalier, and B. Picqué, 2004: Critical slowing down at a bifurcation. Amer. J. Phys., 72, 799-809, doi:10.1119/1.1688783.

Turelli, M., 1977: Random environments and stochastic calculus. Theor. Popul. Biol., 12, 140-178, doi:10.1016/ 0040-5809(77)90040-5.

Wagner, T. J. W., and I. Eisenman, 2015: How climate model complexity influences sea ice stability. J. Climate, 28, 39984014, doi:10.1175/JCLI-D-14-00654.1.

Wong, E., and M. Zakai, 1965: On the convergence of ordinary integrals to stochastic integrals. Ann. Math. Stat., 36, 15601564, doi:10.1214/aoms/1177699916. 